\title{
A Euclidean Approach to Eggs and Polycentric Curves
}

\author{
Angelo Alessandro Mazzotti
}

Published online: 10 May 2014

(C) Kim Williams Books, Turin 2014

\begin{abstract}
This paper examines the problem of smoothly connecting two arcs of curves with two more arcs, and presents the application of this procedure to the drawing of a class of egg-shaped curves and to the generation of some open and closed polycentric curves. The paper provides a solid mathematical background as well as 'ruler and compass' constructions, some of them original techniques. The use of basic, although lengthy, Euclidean proof tools suggests these properties and techniques were within reach-if not known-by architects and scientists long before the invention of analytic geometry. The concepts described in this paper are further developed in its second part (Mazzotti, What Borromini Might Have Known About Ovals. Ruler and Compass Constructions, 2014).
\end{abstract}

Keywords Borromini - San Carlo alle Quattro Fontane - Geogebra · Euclidean geometry · Ovals $\cdot$ Eggs $\cdot$ Polycentric curves

\section{Introduction}

Polycentric curves are curves made of circle arcs subsequently smoothly connected. Their use in architecture has been and still is widespread. For centuries it was the easiest way of connecting points with a curve since only the use of a ruler and a compass was needed, and the infinite amount of possibilities made them an interesting tool for decorative purposes [see for example the pictures on pp. 282-285 in Ragazzo's book (2011)]. The closed convex single-symmetry version - the egg-provides a possible shape for ground plans of buildings, while the closed convex double-symmetry version-the oval-has been used extensively

\footnotetext{
A. A. Mazzotti $(\bowtie)$

Istituto di Istruzione Superiore Statale "Di Vittorio-Lattanzio",

Via Teano, 223, 00177 Rome, Italy

e-mail: angelomazzotti@gmail.com
} 
for both horizontal layouts, such as amphitheatres, stadia and church ground plans (see, for example López Moto 2011), as well as vertical layouts, such as domes, arcs and windows (see for example Huerta 2007). Computer Aided Design, when the appropriate background knowledge is used, can today be used to choose among the infinite possible polycentric curves suiting a particular geometric condition.

This work started in 1999 when I was asked by architect Margherita Caputo to help to understand what baroque artist Francesco Borromini (1599-1667) gave himself as a rule when planning the dome of San Carlo alle Quattro Fontane in Rome, back in the first half of the XVIIth century. He appeared to be able to master the oval form even if at the time only the constructions by Sebastiano Serlio (1619) and a few others (López Moto 2011) appear to have been known.

What Felice Ragazzo (1995) had published just a few years earlier-a simple conjecture about ovals, which he also applied to eggs - showed how it is possible to decide, among an infinite number of possibilities, which oval to draw once the measures of the two axes are given. Although the same paper contains a rigorous mathematical proof of this conjecture by Franco Ghione, I thought it interesting to see if it could be proved only by means of Euclidean geometry, the only tool known at the time of Borromini.

More than ten years later I read F. Ragazzo's extensive book (2011) on polycentric curves (PCCs) where, among many other problems concerning PCCs, he applies an extended version of his conjecture on ovals to a class of constructions of PCCs. The Euclidean proof of it for eggs and PCCs is the subject of the present paper, together with original remarks and original constructions deriving from it. The consequent application to ovals and the possibility to classify the consequent constructions given different choices of parameters is the subject of the second part of this research, published separately in this issue. ${ }^{1}$

Ragazzo's conjecture on how to draw all possible ovals inside a rectangle reduces the problem to quarter ovals inside rectangles. It is the following (author translation from the Italian).

The locus of the connection points for the arcs [of a quarter oval (Ed.)] [...] is the circle, which I will call Connection Locus, defined through the following three points [points 1, 2 and 3 on Ragazzo's original drawing (Fig. 1)]

- intersection between the half major axis and the half minor side [of the rectangle inscribing the oval (Ed.)]

- endpoint of the half minor side transported compass-wise onto the major side

- intersection between the half major side and the half minor axis

(Ragazzo 1995)

"Definitions and Properties of Six-Centre Eggs" is devoted to the basics of sixcentre egg shapes. "Polycentric Curves: Definitions and Issues" presents PCCs and the class of problems this research deals with. In "The Main Theorems for Eggs: Properties of the Connection Locus" I prove the main theorem for eggs and properties

\footnotetext{
${ }^{1}$ Angelo Alessandro Mazzotti, What Borromini Might Have Known About Ovals: Ruler and Compass Constructions.
} 




Fig. 1 Inscribing a quarter oval inside a rectangle: Ragazzo's circle through points 1, 2 and 3 and the quarter oval resulting from the choice of a connection point on it. Image (Ragazzo 1995), reproduced by permission

of Ragazzo's Connection Locus (my translation from the Italian). In "Constructing a Class of Six-Centre Eggs" I apply the proven theorem to the construction of a class of six-centre eggs. "The Main Theorem for Polycentric Curves. Constructing a Class of PCCs" is where the main theorem for PCCs is proved and the different constructions deriving from it - both for open and for closed PCCs—are displayed. "Conclusions and Further Developments" is devoted to my further developments on the subject. The "Appendix" contains most of the proofs of the theorems in this paper.

Geogebra software has been used to draw the different constructions and to get ideas on how to prove the theorems. Animation videos of the different constructions are available on the following website www.mazzottiangelo.eu/en/pcc.asp. This paper only deals with two-dimensional objects.

\section{Definitions and Properties of Six-Centre Eggs}

An egg-shape is an oval which has been allowed to be more pointed on one side:

an egg-shape (or 6-centre egg or simply egg) is a non-twisted closed convex curve with one symmetry axis (or simply axis) made of six arcs of circle subsequently smoothly connected, i.e. sharing a common tangent (Fig. 2).

Due to symmetry it is enough to study half an egg, say the top part in our picture, and for future purposes, divide the top trapezoid - formed by the symmetry axis, the two tangents orthogonal to it and the tangent through the middle connecting pointinto two parts, by drawing the line containing the middle connecting point and the two centres of the corresponding circles.

In this respect, using Fig. 3 as reference, I define

- $A$ and $C$ as the points of intersection between the half-egg and the axis

- $K, J, L$ and $N$ as the centres of the sequence of four arcs the half egg is made of, with radii $r_{1}, r_{2}, r_{3}$ and $r_{4}$ 


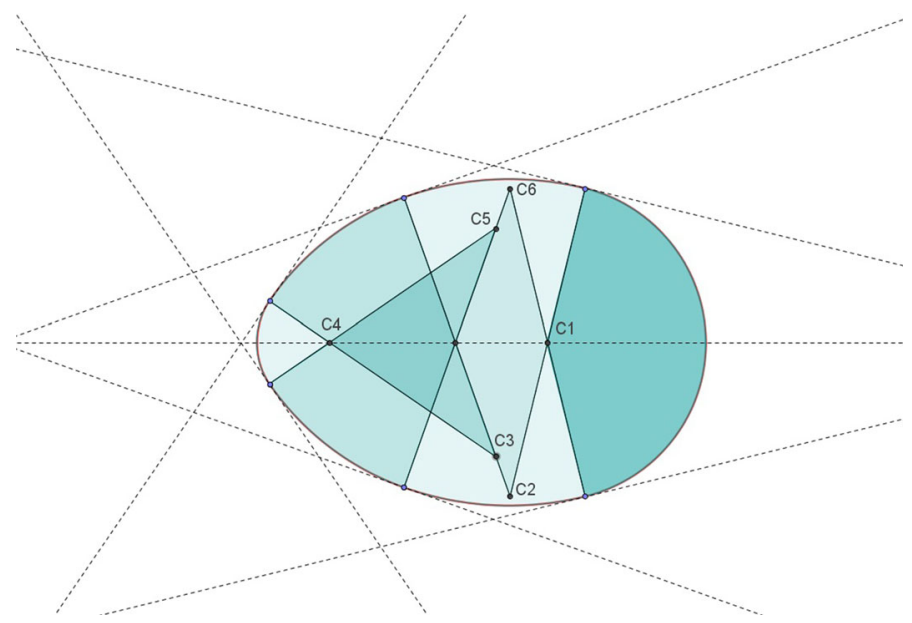

Fig. 2 An egg, with its six centres and the tangents at the connecting points

Fig. 3 A half egg and the two quadrilaterals inscribing it



- $H, B$ and $P$ as the connection points of the pairs of arcs

The above definitions and well-known Euclidean geometry theorems imply that:

- tangents to the circles in $A$ and $C$ are orthogonal to $A C$

- $K$ and $N$ belong to the axis

- the triplets $J \cdot K \cdot H, J \cdot L \cdot B$ and $L \cdot N \cdot P$ are co-linear 
Let $O$ be the intersection of $A C$ with $J B$. If $r_{1}<r_{2}, O$ will lie between $J$ and $B, K$ between $O$ and $A, \overline{O A}>\overline{O B}$ and $\overline{B T}>\overline{A T}$; otherwise (Fig. 3) $J$ will lie between $O$ and $B$ and so on. If $r_{2}=r_{1}$ the curve becomes a four-centre egg.

A string method for designing some multicentre eggs can be found in Dixon (1987: 76).

Because of it's similarity to the problem of inscribing an oval in a rectangle, in "The Main Theorems for Eggs: Properties of the Connection Locus" i will consider, following closely Ragazzo's work (1995), the problem of inscribing a half egg into two quadrilaterals, each with two opposite right angles (in Fig. 3: ATBO and COBS).

\section{Polycentric Curves: Definitions and Issues}

Eggs belong to the family of polycentric curves:

a polycentric curve - from now on $P C C$ - is a curve made of a finite number of arcs of circle subsequently connected with a common tangent in the common point.

This very general definition of a polycentric curve (allowing for twisted and/or non-closed curves), although demanding common tangents in the connecting points, does not require smooth connections. And since the curve is not closed and convex like before, a connection with common tangents is not necessarily smooth. By this I mean that I am also allowing for so-called cusps and flexes made of arcs sharing a common tangent in their connecting point (the three cases are illustrated in Fig. 4). The reason for this is that the theorem which I will prove in "The Main Theorem for Polycentric Curves. Constructing a Class of PCCs" will not have to deal with the different cases separately. On the other hand artists and craftsmen do enjoy choosing among many different possible PCCs, fitting their conditions or constraints, and cusps and flexes represent a wider choice.

Drawing a PCC is straightforward. One starts with an arc of circle (see Fig. 5), draws the normal line at one of the endpoints (for a circle it is the line through the centre) and chooses any point on it as the centre of the next arc, continuing from the endpoint chosen, and so on. What deserves more attention is the case of constructing a PCC subject to constraints. Ragazzo lists five different cases in his book on PCCs (2011: 17), working with circles as well as straight lines and points (circles with infinite or null radius). His case number 4 is the one I tackle in "The Main Theorem for Polycentric Curves. Constructing a Class of PCCs", giving mathematical proof of his method-merely an extension of the theorem on eggs from "The Main Theorems for Eggs: Properties of the Connection Locus"-and presenting constructions made with freeware Geogebra.

The problem is the following:

Given two arcs of circle and a point on each, find all the possible pairs of circles sharing a common tangent in a common point, each one having a common tangent with the given arcs at the given point. 

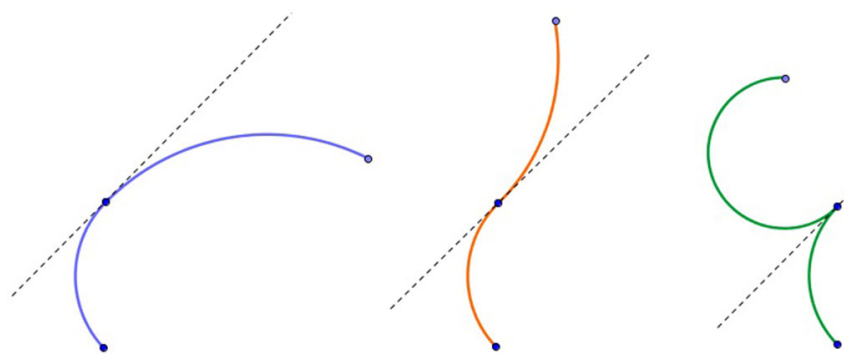

Fig. 4 Three different "common tangent" connections

Fig. 5 Drawing of a PCC

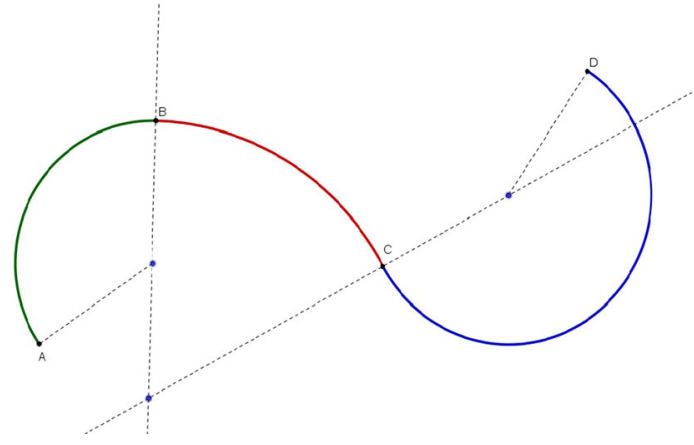

Figure 6 shows the starting arcs, a possible solution and some choices of PCCs obtainable.

\section{The Main Theorems for Eggs: Properties of the Connection Locus}

To be able to inscribe eggs in trapezoids we need to be able to connect arcs of circles.

Given a quadrilateral like $O A T B$ in Fig. 3, with two opposite right angles, how many pairs of circles such as the ones contained in $O A T B$ are there? The answer is: an infinite number, all having something in common. But first of all let's see what happens if I try to impose one of the centres. Anticipating the contents of "Constructing a Class of Six-Centre Eggs", i will construct such a pair of circles extending the XVIIth century construction of Abraham Bosse for an oval (Bosse 1655: 65-66) (described for example by Dotto 2001) also displayed in the following century by Tosca (see López Moto 2011 for references to both original and electronic facsimile), and then show that they are unique.

Let $\overline{B T}>\overline{A T}$.

Choose $K$ between $O$ and $A$. We first determine $F$ along the line $B O$, on the same side of $O$ w.r.t. $B$, such that $\overline{B F}=\overline{A K}$ (see Fig. 7), find $J$ as the intersection of the same line with the axis of the segment $F K$, and then draw the line $J K$; the 

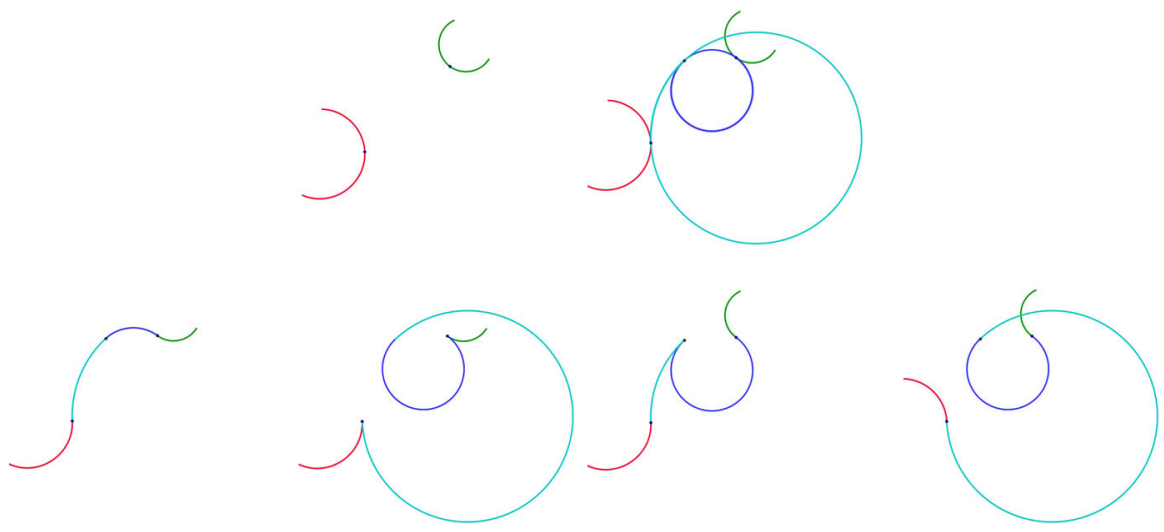

Fig. 6 For any two given arcs and a point on each, two tangent circles are found, each tangent to one of the arcs at the given point. Different choices of arcs selected from this construction give different PCCs

intersection with the circle with centre $K$ and radius $\overline{A K}$ lying inside the quadrilateral is the connection point $H$ for the two arcs, the first being $A H$ with centre $K$, and the second one being $H B$ with centre $J$. The drawn arcs enjoy the desired properties.

On the other hand for any such arcs inscribed in $O A T B$ - part of an egg-with $K$ as one of the centres, the only point $F$ between $J$ and $B$ such that $\overline{F B}=\overline{K A}$, forms with $K$ and $B$ the same angle as in the above construction, and thus with $K$ and $J$ the same isosceles triangle. The two drawings have to be the same.

Note that in order for the axis of $F K$ to meet $B O, B \hat{F} K$ has to be obtuse, i.e.

$$
\overline{O F}<\overline{O K} \cdot \cos (\pi-A \hat{O} B)=-\overline{O K} \cdot \cos A \hat{O} B
$$

which can be written

$$
\overline{B F}-\overline{O B}=\overline{A K}-\overline{O B}=\overline{A O}-\overline{O K}-\overline{O B}<-\overline{O K} \cdot \cos A \hat{O} B .
$$

Solving the last inequality w.r.t. $\overline{O K}$ we get the following condition for $K$

$$
\overline{O K}>\frac{\overline{O A}-\overline{O B}}{1-\cos A \hat{O} B} .
$$

I first wrote the following theorem for ovals, proving Ragazzo's conjecture (Ragazzo 1995) via Euclidean geometry, and then succeeded in extending it to eggs. The following form allows for an extension to polycentric curves-as will be shown in "The Main Theorem for Polycentric Curves. Constructing a Class of PCCs" — as well as the application to ovals - as shown in the already cited second part of this paper.

Theorem 1 Let $O A T B$ be a quadrilateral with $\overline{B T}>\overline{A T}$ and opposite $T \hat{B} O$ and $\hat{O A T}$ right angles (see Fig.8). Let $A \hat{O} B=\alpha$. Let $S$ be the point on BT such that $\overline{S T}=\overline{A T}$. Necessary and sufficient condition for $H$ to be the connecting point for two arcs of circle with centres inside $B \hat{T} A$ and tangent in $H$, one tangent to the line $A T$ in $A$ and the other tangent to the line $T B$ in $B$, is for $H$ to belong to the open arc 

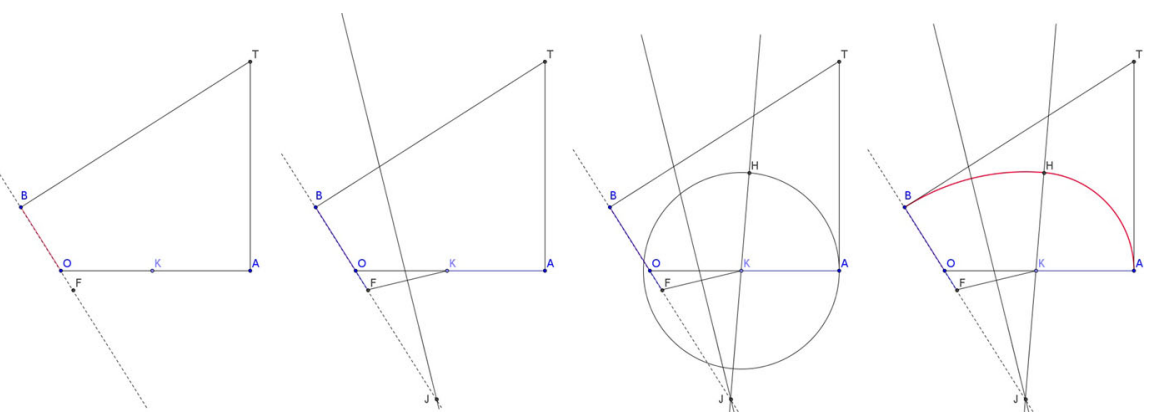

Fig. 7 Inscribing two arcs into a quadrilateral having two opposite right angles, extending Bosse's method for ovals, starting from $K$ when $O B<O A$

AS of the circle through A, B and S. Moreover, the angle with origin at the centre of such a circle corresponding to the arc $A B$ is equal to $\alpha$.

If $H \equiv S$ or $H \equiv A$ the arcs in Theorem 1 cannot be built.

Proof See "Appendix" at the end of this paper.

Note that, since $\overline{A V}=\overline{V S}$, as is easily proved, the line $R V$ bisects both concave and convex angles $A \hat{V} S$, forming a pair of $\frac{\alpha}{2}$ angles on the convex side, therefore passing through point $T$. Moreover $R K$ bisects $A \hat{K} H$, because triangles $A K R$ and $R K H$ are equal. But triangles $J R H$ and $J R B$ are also equal, therefore $J R$ bisects $K \hat{J} O$.

Bosse's construction, the limitation for point $K$ and Theorem 1 have been made and proved in the case $\overline{B T}>\overline{A T}$. If on the other hand the side of the quadrilateral on the symmetry axis of the egg is smaller than the one on the normal to the middle tangent point, everything works exchanging $J$ with $K$ and $A$ with $B$. Bosse's construction can start from a point $J$ between $O$ and $B$ (see Fig. 9), for which the limitation $\overline{O J}>\frac{\overline{O B}-\overline{O A}}{1-\cos A \hat{O B}}$ can be proved (although for the purpose of drawing we could again start from $K$ on the other side of $A$ w.r.t. $O$ ), and the whole of Theorem 1 works in exactly the same manner, since the above exchange of point names-after having mirrored Fig. 8 w.r.t. the bisector of angle $\alpha$ (see Fig. 10)-leaves substance unchanged. I'll reproduce the changed version, for the sake of completeness.

Theorem 2 Let $O A T B$ be a quadrilateral with $\overline{A T}>\overline{B T}$ and opposite $T \hat{B} O$ and $O \hat{A} T$ right angles (see Fig.10). Let $A \hat{O} B=\alpha$. Let $S$ be the point on $A T$ such that $\overline{S T}=\overline{B T}$. Necessary and sufficient condition for $H$ to be the connecting point for two arcs of circle with centres inside $B \hat{T} A$ and tangent in $H$, one tangent to the line $A T$ in $A$ and the other tangent to the line $T B$ in $B$, is for $H$ to belong to the open arc $B S$ of the circle through $A, B$ and $S$. Moreover, the angle at the centre of such $a$ circle corresponding to the arc $A B$ is equal to $\alpha$.

Finally, if $\overline{B T}=\overline{A T}, K$ and $J$ coincide with $O$ and the two arcs become a single one. 


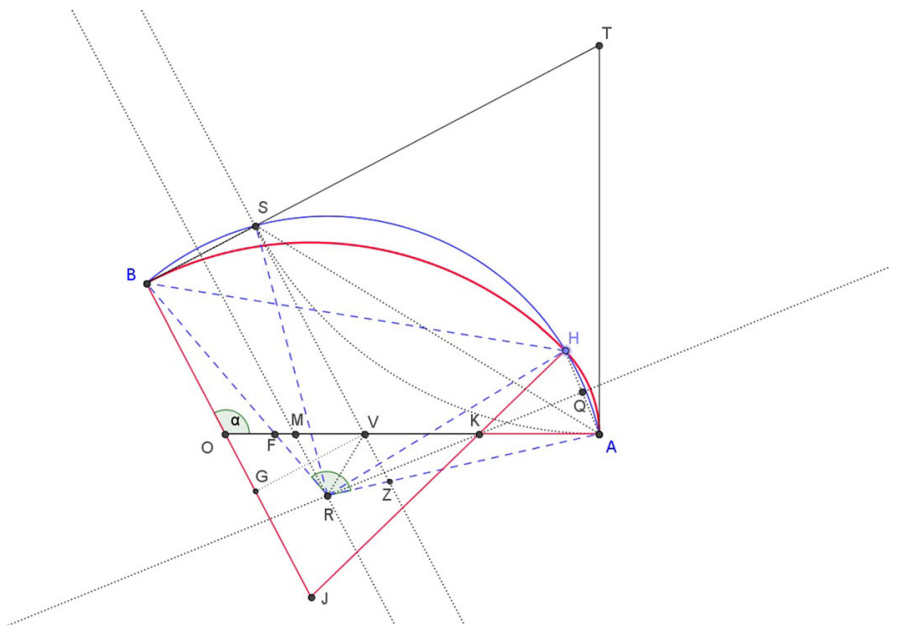

Fig. 8 The construction of the egg arcs in Theorem 1

Fig. 9 Inscribing two arcs into a quadrilateral with two opposite right angles extending Bosse's method for ovals, starting from $J$ when $O A<O B$

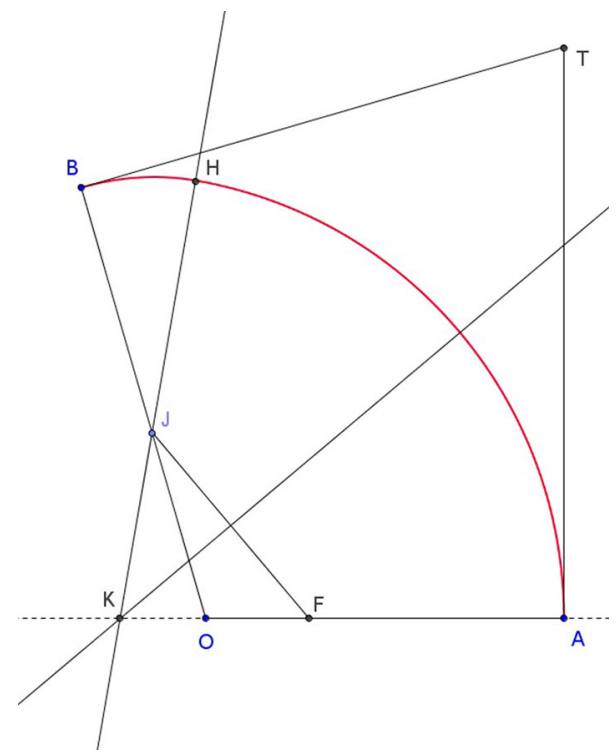

Definition I will call the circle through $A, B$ and $S$, following Ragazzo's work (1995), from now on Connection Locus (my translation), or simply CL.

Summing up the properties of point $R$ for quarter eggs we have the following.

Theorem 3 The centre $R$ of the Connection Locus for a quarter egg is the incentre of triangle $O K J$.

The fact that $R O$ bisects $J \hat{O} A$ (or its adjacent angle) is another result already conjectured by Ragazzo (1995). 
Fig. 10 The construction for Theorem 1 corresponding to the case $O A<O B$

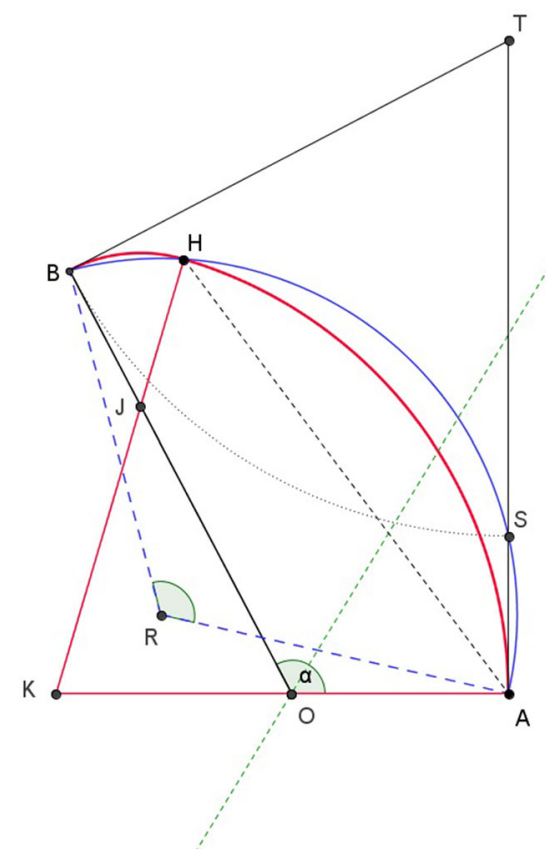

\section{Constructing a Class of Six-Centre Eggs}

Six-centre eggs can be constructed in different ways. They have 9 of freedom, although with some constraints. Take for example four points on a plane (equivalent to eight parameters) and then fix the measure of one or the radii.

Construction of a (six-centre) egg given four consecutive centres and the radius of the first one. For example points $A, B, C$ and $D$ and measure $r$ in Fig. 11 (see link to Geogebra animation video on my website www.mazzottiangelo.eu/en/pcc.asp):

- draw the line $A D$

- draw the circle with centre $A$ and radius $r$, let $M$ be the intersection with $A D$

- find point $F$ as the intersection between this first circle and the line $A B$

- draw the circle with centre $B$ and radius $\overline{B F}$ and find its intersection $H$ with $B C$

- draw the circle with centre $C$ and radius $\overline{C H}$ and find its intersection $I$ with $C D$

- draw the circle with centre $D$ and radius $\overline{D I}$ and find its intersection $L$ with $A D$

- mirror arcs $M F, F H, H I$ and $I L$ w.r.t. $A D$ to get the whole egg.

It is important to stress that not all combinations of four points and one radius do create a six-centre egg: intersections between arcs and centre lines have to be on the right side. It is also true that the same set of points and radius can yield a different oval if points are chosen in a different order. See Fig. 12, where I have exchanged the roles of $B$ and $C$ and compare it to the egg in Fig. 11. The class of six-centre eggs I am interested in is that which can be built using the CL. That is, I will consider here the problem of inscribing a six-centre egg inside a given isosceles trapezoid, once the tangent point(s) to the oblique side is (are) given. Ragazzo has 




Fig. 11 Constructing an egg given 4 consecutive centres of non-symmetrical circles and a radius

already solved the problem since the CL is his own invention (see Ragazzo 1995, 2011) although he has not given proof of his conjecture. I will repeat the construction here in a more systematic way referring directly to Theorem 1, which is my own original contribution, dealing also with situations not yet tackled, in order to solve the problem completely.

The question is:

Given an isosceles trapezoid and two symmetric points on the oblique sides, is it always possible to inscribe a six-centre egg with the same symmetry axis, tangent to the parallel sides with two opposite arcs, and tangent to the oblique sides at the given points exactly where each of the other two pairs of arcs connect? How many are there for any given trapezoid?

The procedure should at this point be clear, but one important issue has not been tackled yet. Given an isosceles trapezoid, we draw its symmetry axis and then choose a point on one of the oblique sides. From there we draw the perpendicular to the oblique side itself, dividing the half trapezoid into two quadrilaterals (OATB and $O B S C$ in Fig. 13a) satisfying the conditions of Theorems 1 and 2. That is unless this perpendicular intersects the symmetry axis outside segment $\overline{A C}$ (see Fig. 13b). We need to find out if the two tangent arcs of circle needed inside the trapezoid with the usual properties can still be drawn in the latter case, and that they are unique.

The answer is yes. And the proof is the same as that of Theorem 1, although a few changes need to be made.

Theorem 4 Let OATB be a tangled quadrilateral (see Fig.14) with BT and AO crossing each other, $\overline{B T}>\overline{A T}$ and opposite TBOAnd OÂT right angles. Let $\alpha$ be the 


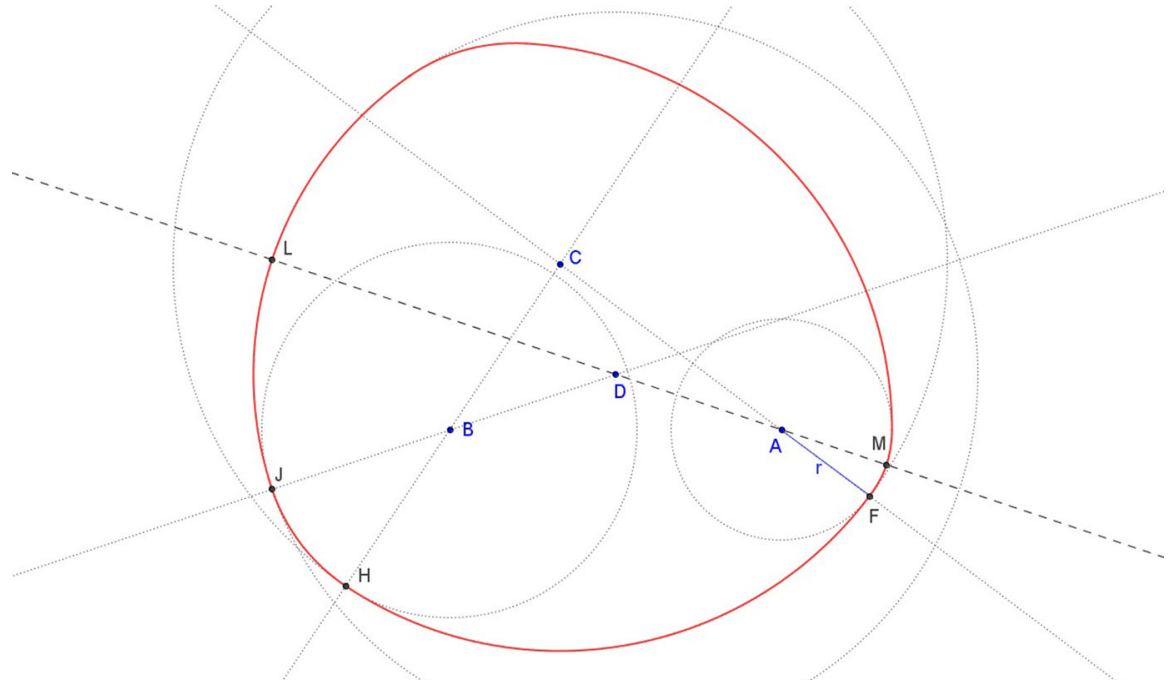

Fig. 12 Constructing a different egg given the same four consecutive centres of non-symmetrical circles and the same radius

external angle to $A \hat{O} B$. Let $S$ be the point on BT such that $\overline{S T}=\overline{A T}$. Necessary and sufficient condition for $H$ to be the connecting point for two arcs of circle with centres inside BTA and tangent in $H$, one tangent to the line AT in A and the other tangent to the line $T B$ in $B$, is for $H$ to belong to the open arc AS of the circle through $A, B$ and $S$. Moreover, the angle at the centre of such a circle corresponding to the arc $A B$ is equal to $\alpha$.

Proof See "Appendix".

Thanks to Theorem 4 I can now say that any point $B$ can be chosen on segment $S T$ in Fig. 13 because a CL can be drawn on both sides of $B O$ and on each of the two a connection point freely chosen. I can now state that in any given isosceles trapezoid (at least) $\infty^{3}$ six-centre eggs can be inscribed. This is how it is done.

Construction of a (six-centre) egg inscribed in a given isosceles trapezoid (see Fig. 15 and link to Geogebra animation video on my website www.mazzottiangelo. eu/en/pcc.asp).

- given the isosceles trapezoid $C^{\prime} D^{\prime} T S$ of bases $C^{\prime} S$ and $T D^{\prime}$, draw the symmetry axis $A C$ and choose a point $B$ on the oblique side $T S$ to be the middle connection point

- draw from $B$ the orthogonal line to $S T$ and let $O$ be the intersection with the line $A C$

- since $\overline{B T}<\overline{A T}$ find the point $S^{\prime}$ on segment $A T$ such that $\overline{T S^{\prime}}=\overline{B T}$

- since $\overline{B S}>\overline{S C}$ find the point $S^{\prime \prime}$ on segment $S B$ such that $\overline{S S^{\prime \prime}}=\overline{S C}$

- draw the $\operatorname{arc} B S^{\prime}$ of the CL-with centre $R$ - $\operatorname{through} A, S^{\prime}$ and $B$, and the $\operatorname{arc} C S^{\prime \prime}$ of the CL-with centre $R^{\prime}$ - through $C, S^{\prime \prime}$ and $B$

- choose $H$ on $\operatorname{arc} B S^{\prime}$ and $P$ on $\operatorname{arc} C S^{\prime \prime}$ 


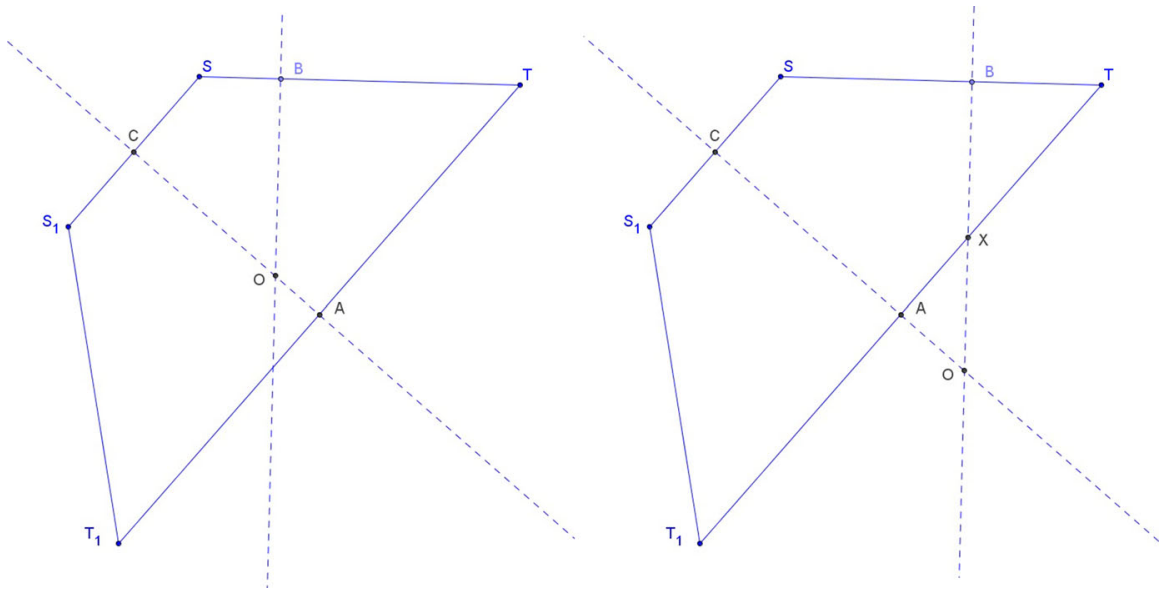

Fig. 13 Inscribing an egg inside a trapezoid. Two different situations

Fig. 14 Inscribing two tangent arcs inside a tangled quadrilateral

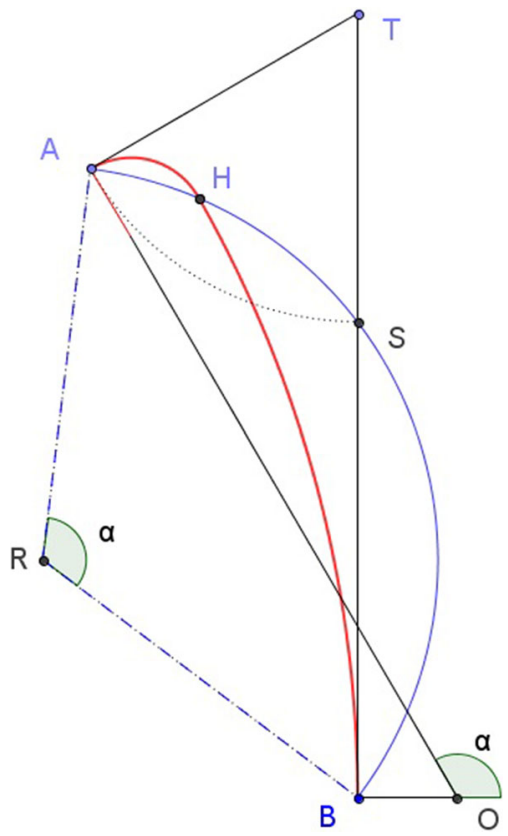

- the axis of segment $A H$ intersects $O A$ in point $K$, the axis of $H B$ intersects $B O$ in point $J$, the axis of $P B$ intersects $B O$ in point $L$, the axis of segment $P C$ intersects $A C$ in point $N$

- $\operatorname{arcs} A H$ with centre $K, H B$ with centre $J, B P$ with centre $L$ and $P C$ with centre $N$, and their symmetric arcs, form the desired egg 


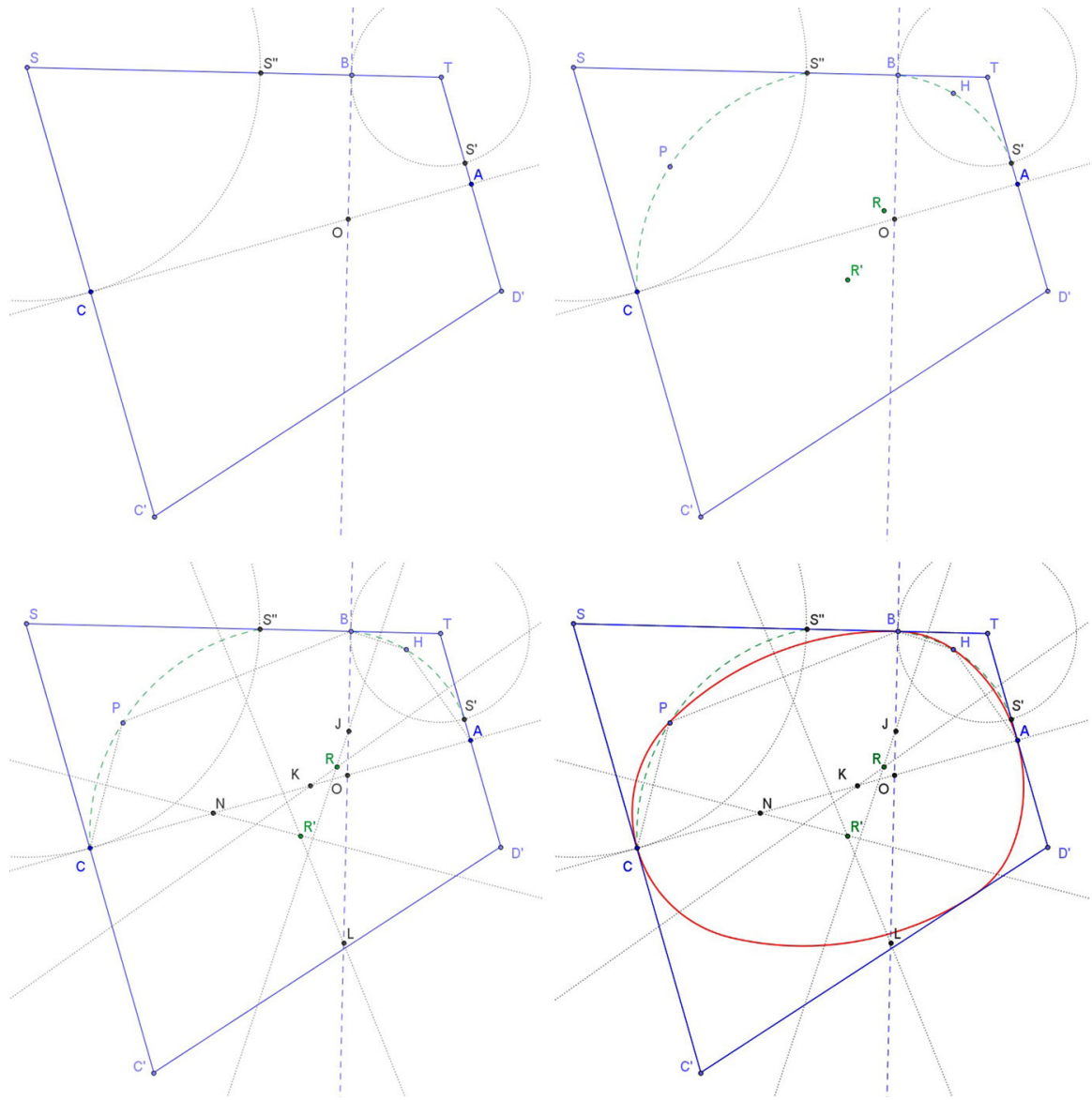

Fig. 15 Constructing a four-centre egg inscribed in an isosceles trapezoid

\section{The Main Theorem for Polycentric Curves. Constructing a Class of PCCs}

As I already pointed out, eggs are an extension of ovals, and both are a very special case of polycentric curves (PCCs). Because of their increased complexity, there are many different approaches to the problem of finding the PCC satisfying this or that condition. Felice Ragazzo's book tackles and solves five of these problem (see Ragazzo 2011: 17) including the one I talk about in this sectionfor which he illustrates the whole of the different possibilities of resulting PCCs depending on the mutual position of the given circles (Ragazzo 2011: 146-213). My contribution to the subject is the mathematical proof of the main construction tool and of other properties of the CL involved, as well as some new remarks and the possibility of viewing the constructions I made with Geogebra. 




Fig. 16 The system of tangents with base points $A$ and $B$

As stated in "Polycentric Curves: Definitions and Issues", the problem is:

Given two arcs of circle and a point on each, find all the possible pairs of different circles sharing a common tangent in the common point, each one having a common tangent with the given arcs at the given point.

I will tackle the problem by means of extending Theorem 1 to the case of PCCs. Before this, I need to sum up, or prove, all the properties enjoyed by a special quadrilateral and by the two circles that one derives from it, forming together the CL. The inspiration for the following approach was Ragazzo's drawings (Ragazzo 2011: 131-145).

\section{Systems of Two Tangents}

Consider the following as being a system of two tangents with base points $A$ and $B$ (see Fig. 16).

Given two points $A$ and $B$ on a plane and two non-parallel lines through them $t_{A}$ and $t_{B}$ - thought of as tangents in $A$ and $B$ to curves - draw the perpendiculars from $A$ and $B, n_{A}$ and $n_{B}$. Let $O=n_{A} \cap n_{B}$ and $T=t_{A} \cap t_{B}$. Let's build the CL; let:

$S_{1}$ and $S_{4}$ be the points on $T B$, the first one on $B$ 's side the second on $T$ 's side, such that $\overline{T S_{1}}=\overline{T S_{4}}=\overline{T A}$

$S_{2}$ and $S_{3}$ be the points on $T A$, the first one on $A$ 's side the second on $T$ 's side, such that $\overline{T S_{2}}=\overline{T S_{3}}=\overline{T B}$

$\alpha$ the obtuse angle formed by $n_{A}$ and $n_{B}$ and $\beta=\pi-\alpha$ the acute angle formed by $t_{A}$ and $t_{B}$

Find the centre $R$ of the circle $\Gamma_{1}$ through $A, B$ and $S_{2}$ just by intersecting the axis of segment $A B$ and the axis of segment $B S_{2}$, which is also the bisector of $A \hat{T} B$. Now 


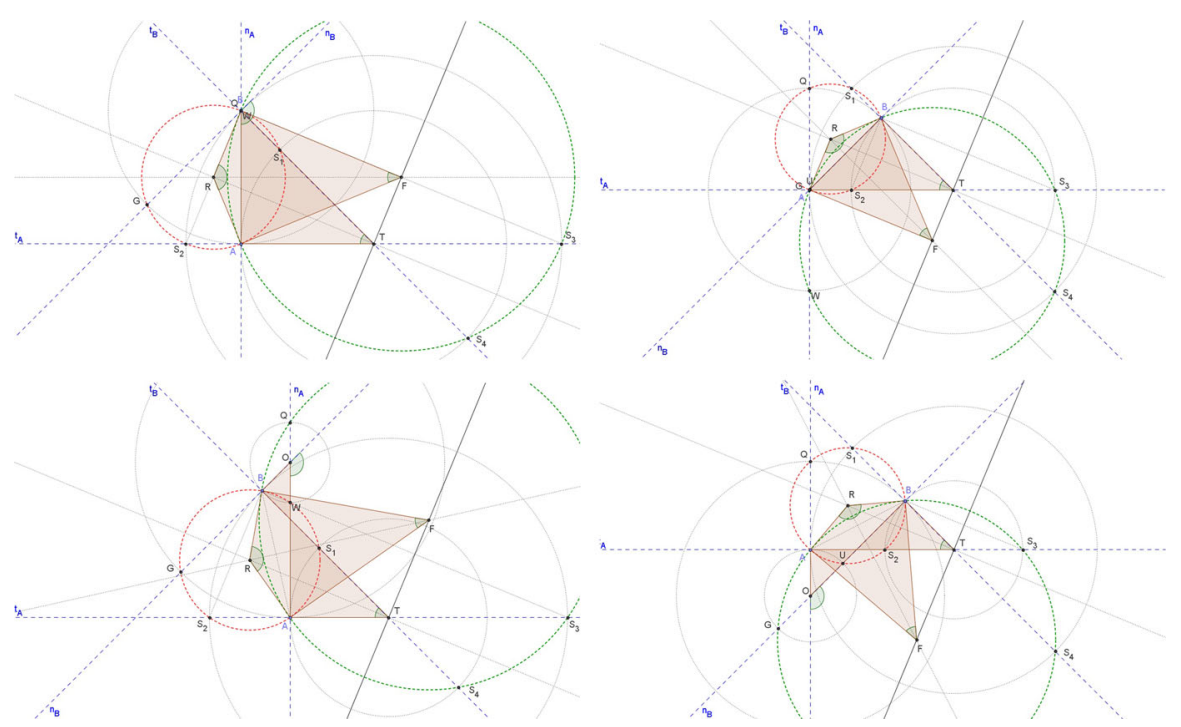

Fig. 17 Different forms for a system of tangents with two base points $A$ and $B$

note that the two equal triangles $T R B$ and $T R S_{2}$ are also formed, and so $S_{2}$ has also to be on $\Gamma_{1}$. The same can be shown for the second circle, $\Gamma_{2}$ the circle with centre $F$ through $A, B, S_{3}$. Let's check the angles in the quadrilateral $A F B R$ : since $\overline{S_{1} T}=\overline{A T}$ one has that $T \hat{S}_{1} A=\frac{\pi-\beta}{2}=\frac{\alpha}{2}$, which implies that $R \hat{S}_{1} A+R \hat{S}_{1} B=A \hat{S}_{1} B=\pi-\frac{\alpha}{2}$ and so, since $R \hat{S}_{1} A=R \hat{A} S_{1}$ and $R \hat{S}_{1} B=R \hat{B} S_{1}$, that $R \hat{A} S_{1}+R \hat{B} S_{1}=\pi-\frac{\alpha}{2}$; looking at the quadrilateral $R A S_{1} B$ we can deduce that

$$
A \hat{R} B=2 \pi-\left(\pi-\frac{\alpha}{2}\right)-\left(\pi-\frac{\alpha}{2}\right)=\alpha .
$$

It is similarly proved that $B \hat{F} A=\beta$, and thus that $R \hat{B} F$ and $R \hat{A} F$ have to be right angles. We finally notice that $F T S_{3}$ and $F T B$ are equal therefore $T F$ bisects $B \hat{T} S_{3}$.

All these remarks stay true when $O \equiv A$ or $O \equiv B$ and $O A T B$ becomes a triangle, or even when $O A T B$ becomes tangled, as can be shown by checking the above properties on the different cases in Fig. 17.

Summing up, this means that centres $R$ and $F$ of $\Gamma_{1}$ and $\Gamma_{2}$ can be found simply intersecting the axis of $A B$ with the two bisectors of the angles in $T$ formed by $t_{A}$ and $t_{B}$. Furthermore $R A F B$ shares the same angles as $A O B T$ when it is not tangled.

The Main Theorem

We now need a whole new theorem to show that $\Gamma_{1}$ and $\Gamma_{2}$ actually form the CL and draw our polycentric curves. The use of analytic geometry to prove this same theorem would probably make things much more straightforward. My choice of using Euclidean geometry is that of remaining consistent with the whole setup of the present work. 




Fig. 18 Constructing circles tangent to a given one and to a given straight line on a given point

Theorem 5 Given a system of tangents with base points $A$ and $B$, for any $H \in \Gamma_{1} \cup \Gamma_{2}-\left\{A, B, S_{1}, S_{2}, S_{3}, S_{4}\right\}$ (see Fig.16) there exist two circles $\Gamma_{3}$ and $\Gamma_{4}$ tangent with each other in $H$, such that $t_{A}$ is tangent to $\Gamma_{3}$ in $A$ and $t_{B}$ is tangent to $\Gamma_{4}$ in $B$.

\section{Proof See “Appendix".}

I now need to prove the reverse of Theorem 5. In order to do so I have to show how all pairs of tangent circles, one with tangent $t_{A}$ in $A$ and the other with tangent $t_{B}$ in $B$, can be detected. I will use the following construction, which I found in Ragazzo's book (2011), and then prove by means of Lemma 7 that such a construction is what one needs to find all possible circles tangent to a given one and tangent in $A$ to a straight line through $A$.

Let $A$ be a point, $t_{A}$ a straight line through it and $\Gamma_{4}$ a circle with centre $J$ (see Fig. 18).

let $n_{A}$ be the perpendicular to $t_{A}$ in $A$ and $E$ and $F$ the intersections of the parallel to it through $J$ with $\Gamma_{4}$

let $H_{1}$ and $H_{2}$ be the new intersections of $F A$ and $E A$ with $\Gamma_{4}$

let $K_{1}$ and $K_{2}$ be the intersections of $J H_{2}$ and $J H_{1}$ with $n_{A}$

Triangles $K_{1} H_{1} A$ and $F J H_{1}$ are similar, therefore $\overline{K_{1} A}=\overline{K_{1} H_{1}}$ and the circle with centre $K_{1}$ and radius $\overline{K_{1} A}$ passes through $H_{1}$ and is tangent to $\Gamma_{4}$ since $H_{1}, K_{1}$ and $J$ are co-linear. Moreover triangles $K_{2} H_{2} A$ and $E J H_{2}$ are also similar, therefore $\overline{K_{2} A}=$ $\overline{K_{2} H_{2}}$ and the circle with centre $K_{2}$ and radius $\overline{K_{2} A}$ passes through $H_{2}$ and is tangent to $\Gamma_{4}$ since $H_{2}, K_{2}$ and $J$ are co-linear.

If $\Gamma_{4}$ is tangent to $t_{A}$ then one of the circles becomes a straight line, while if $A \in \Gamma_{4}$ no circle can be drawn. 
Lemma 6 Any circle tangent to a given circle $\Gamma_{4}$ and to a given straight line $t_{A}$ in a given point $A$ can be found by means of the above procedure.

Proof See "Appendix".

Knowing how to find all connection points $H$ we can prove the property they enjoy.

Theorem 7 Given a system of tangents with base points $A$ and $B$, for any circles $\Gamma_{3}$ and $\Gamma_{4}$ tangent in $H$, such that $t_{A}$ is tangent to $\Gamma_{3}$ in $A$ and $t_{B}$ is tangent to $\Gamma_{4}$ in $B$, we have that $H \in \Gamma_{1} \cup \Gamma_{2}-\left\{A, B, S_{1}, S_{2}, S_{3}, S_{4}\right\}$.

Proof See "Appendix".

Keeping in mind that two points on two smooth curves define a system of tangents as described in "Systems of Two Tangents", we can finally state the main theorem.

Theorem 8 Given two arcs (of circle) and a point on each-A and B-such that the tangents through these points are not parallel, necessary and sufficient condition for $H$ to be the connecting point for a pair of tangent circles, each tangent to one of the arcs in the given point, is for $H$ to belong to $\Gamma_{1} \cup \Gamma_{2}-\left\{A, B, S_{1}, S_{2}, S_{3}, S_{4}\right\}$.

Proof A direct consequence of Theorems 5 and 7.

We just need to prove that, as Ragazzo realised, the CL for two arcs and two points on them such that the tangents are parallel, is simply the line through these two points leaving those points out.

Theorem 9 Given two arcs (of circle) and a point on each-A and B-such that the tangents through these points are parallel (see Fig.19), necessary and sufficient condition for $H$ to be the connecting point for a pair of tangent circles, each tangent to one of the arcs in the given point, is for $H$ to belong to $A B-\{A, B\}$.

Proof See “Appendix".

Joining Two Arcs with Two Arcs

We are finally ready to draw our polycentric curves. Given any pair of arcs of curve (or even segments) with a point on each- $A$ and $B$-where a single tangent is defined, we construct the system of tangents described in "Systems of Two Tangents", finding $R$ and $F$ as the intersection between the axis of $A B$ and the two bisectors of the angles in $T$ (see Fig. 20). The circles $\Gamma_{1}$ and $\Gamma_{2}$ through $A$ and $B-$ with centres $R$ and $F$ - are the CL, once we have crossed out points $S_{1}, S_{2}, S_{3}, S_{4}$, as well as $A$ and $B$, as proved by Theorem 8 (see link to Geogebra animation at www. mazzottiangelo.eu/en/pcc.asp).

Take any point $H$ on $\Gamma_{1}-\left\{A, B, S_{1}, S_{2}\right\}$ and draw the circle through $A$ and $H$ having its centre $K$ on line $O A$, then find $J$ as the intersection between lines $H K$ and $O B$ and draw the circle with centre $J$ and radius $\overline{J H}$. We are now free to choose any of the sixteen possible four-arc polycentric curves through $A, H$ and $B$ just by means 




Fig. 19 The CL (the broken line) for a system of parallel tangents through $A$ and $B$

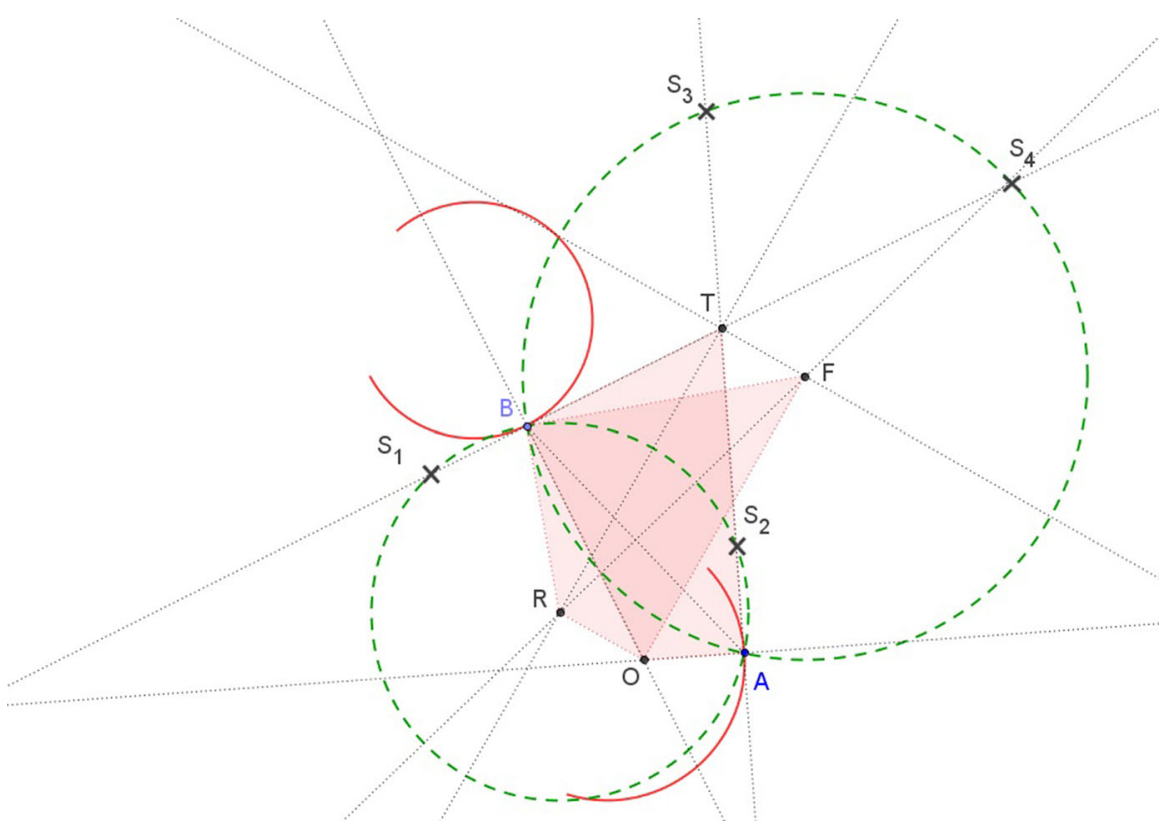

Fig. 20 The CL given two arcs of circle and a point on each $-A$ and $B$

of deciding which arcs to keep. Figure 21 shows - for a choice of $\mathrm{H}$-four possible choices of arcs (see link to Geogebra animation video on my website www. mazzottiangelo.eu/en/pcc.asp). 


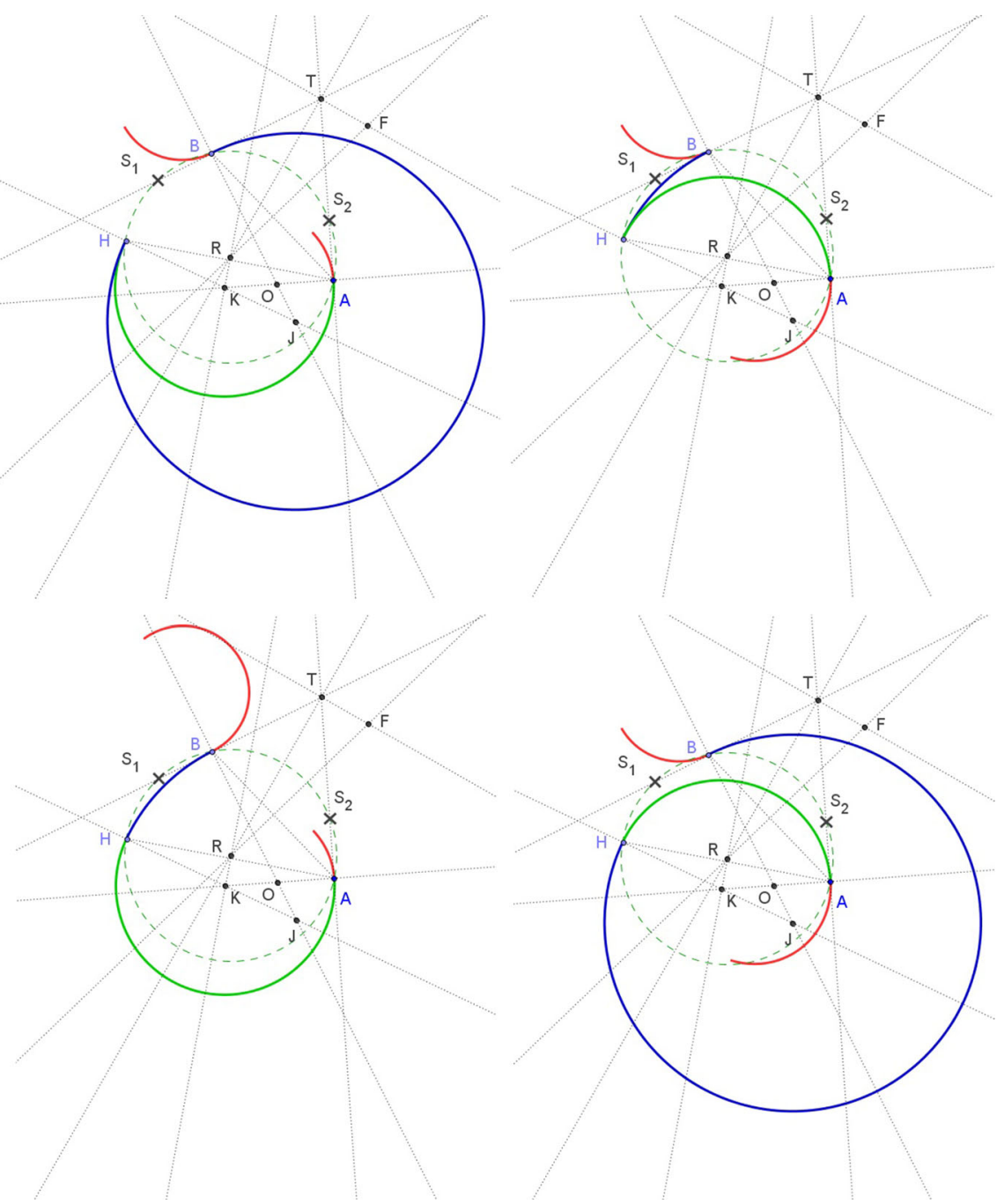

Fig. 21 For a choice of $H$ on $\Gamma_{1}-\left\{A, B, S_{1}, S_{2}\right\}$, four possible choices of a polycentric curve

The other possibility is of course to choose $H$ on $\Gamma_{2}-\left\{A, B, S_{3}, S_{4}\right\}$, repeating the same procedure (Fig. 22; see link to Geogebra animation video at www. mazzottiangelo.eu/en/pcc.asp).

Choosing $H$ to be $S_{1}, S_{2}, S_{3}$ or $S_{4}$ is also possible, when one is ready to accept polycentric curves including segments.

Parallel tangents can also yield four-arc polycentric curves. The construction is a straightforward application of Theorem 9. 


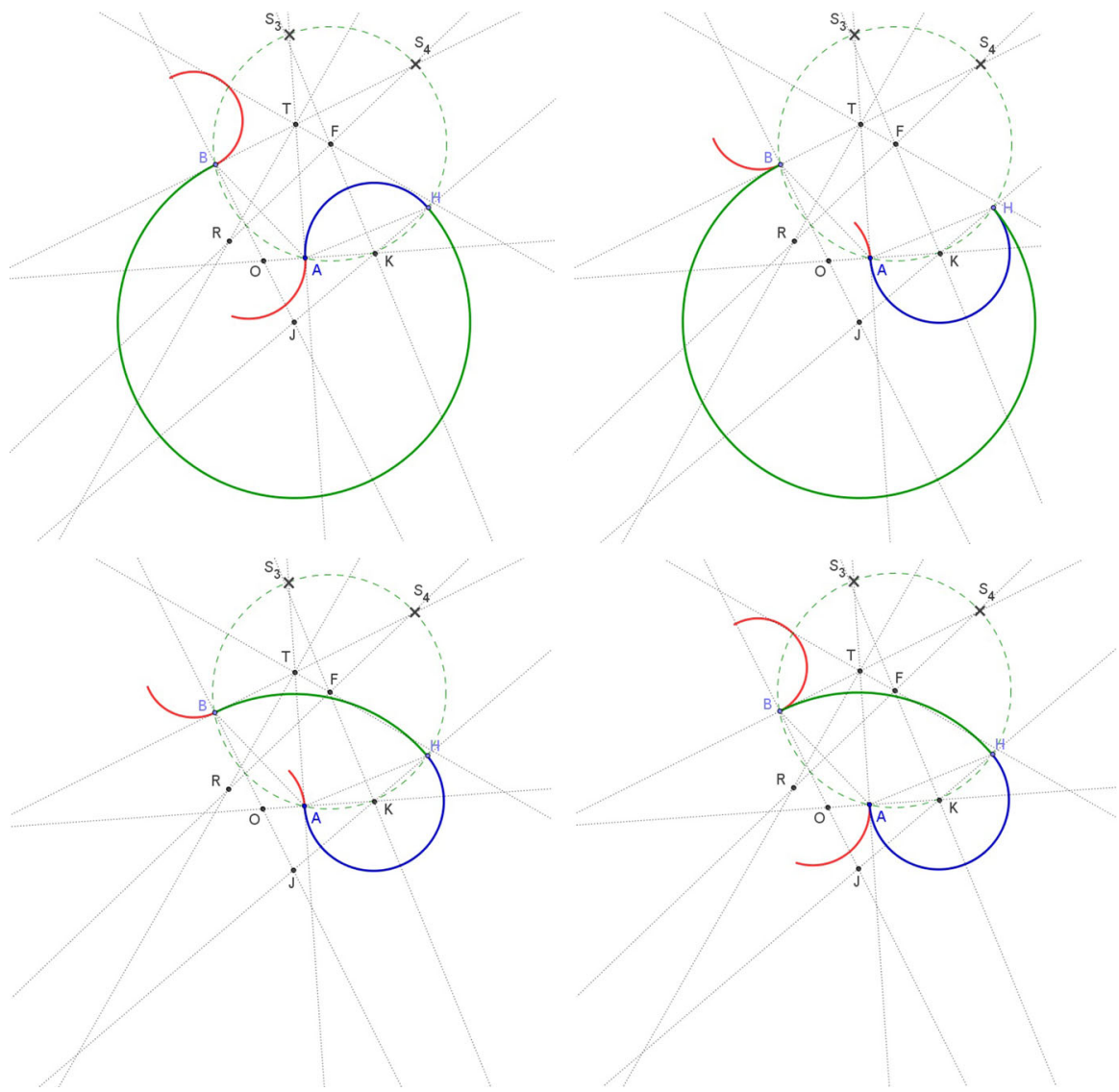

Fig. 22 For a choice of $H$ on $\Gamma_{2}-\left\{A, B, S_{3}, S_{4}\right\}$, four possible choices of a polycentric curve

\section{Four-arc Closed PCCs}

Given two points, $A$ and $B$, and defining two lines through $A$ and $B$, it is also possible to draw four-arc closed polycentric curves with them as first and third connecting points and the given lines as tangents. It is enough to repeat the procedure to draw the four-arc polycentric curve, this time choosing two $H$ points. In this way we will have two sets of two $\operatorname{arcs}$ starting and ending in $A$ and $B$, that is a four-arc closed polycentric curve. Figure 23 shows four possible choices of two points $H$ and $H_{1}$ and for each a choice (among sixteen) of arcs (see link to Geogebra animation at www.mazzottiangelo.eu/en/pcc.asp).

\section{Conclusions and Further Developments}

Mathematical rigour and practical drawing applications are the topics of this paper. The freeware Geogebra has been crucial to achieve both. Furthermore, only Euclidean geometry has been used throughout this work. 


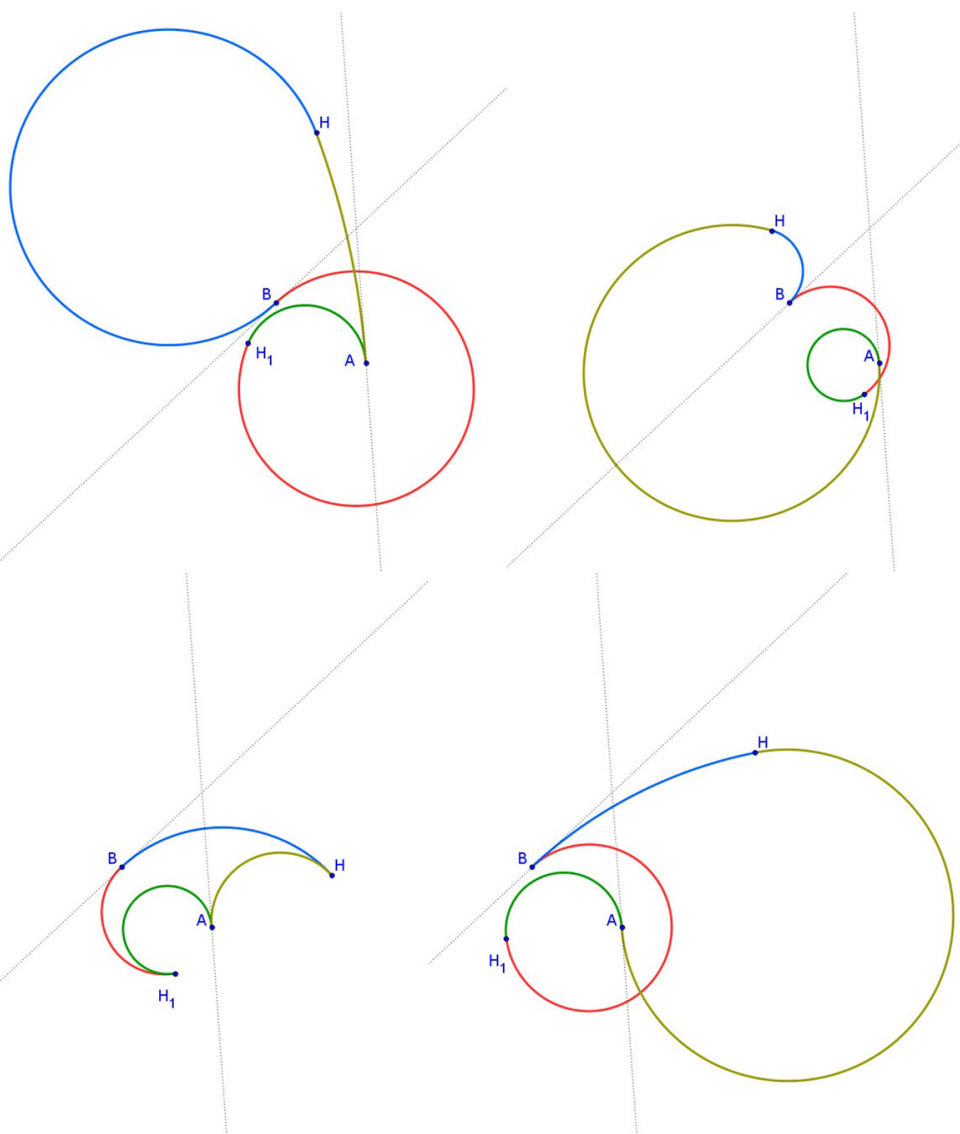

Fig. 23 Four possible four-arc closed PCCs given two alternate connecting points $A$ and $B$ and their tangents

The consequences for the constructions of ovals, and an attempt to classify them, as well as a conjecture on the role they might have played in Baroque architecture, are the topics of the second part of this paper in the present issue of this journal (Mazzotti 2014).

An analytic approach to the study of ovals, eggs and PCCs will be the subject of a future paper.

Acknowledgments I would like to thank Stefano Herzel for his friendship, help and encouragement, Felice Ragazzo for the long talks and the exchange of ideas and Sophie Püschmann for her constant support.

\section{Appendix to a Euclidean Approach to Eggs and Polycentric Curves}

\section{Proof of Theorem 1}

I will start from the second part of the theorem. Let $R$ be the centre of the circumference through $A, B$ and $\mathrm{S}$; since $\overline{S T}=\overline{A T}$ one has that $T \hat{S} A=\frac{\pi-(\pi-\alpha)}{2}=\frac{\alpha}{2}$, 
which implies that $R \hat{S} A+R \hat{S} B=A \hat{S} B=\pi-\frac{\alpha}{2}$ and so, since $R \hat{S} A=R \hat{A} S$ and $R \hat{S} B=R \hat{B} S$, that $R \hat{A} S+R \hat{B} S=\pi-\frac{\alpha}{2}$; looking at the quadrilateral $R A S B$ we can deduce that

$$
A \hat{R} B=2 \pi-\left(\pi-\frac{\alpha}{2}\right)-\left(\pi-\frac{\alpha}{2}\right)=\alpha
$$

For the necessary condition I will show that two generic arcs of circumference enjoying the properties stated have their connection point on the described circumference. If $\mathrm{K}$ on $\mathrm{OA}$ is the centre of one of the two, we have (see previous remarks in "The Main Theorems for Eggs: Properties of the Connection Locus", as well as the properties of six-centre eggs in "Definitions and Properties of Six-Centre Eggs", when $\overline{B T}>\overline{A T}$ ) that $\frac{\overline{O A}-\overline{O B}}{1-\cos \alpha}<\overline{O K}<\overline{O A}$.

The connection point belongs to the circumference with centre $\mathrm{K}$ and radius $\overline{K A}$. I will prove that it is the other intersection $H$ between this circumference and the one through $A, B$ and S. Having detected intersection point $J$ of $K H$ with $O B$, if one proves that $\overline{J H}=\overline{J B}$ then the second circumference uniquely determined by $A, B$ and $K$ (see the remarks in "The Main Theorems for Eggs: Properties of the Connection Locus"), having centre in $\mathrm{J}$ and radius equal to $\overline{B J}$, will be found; the connection point of the two arcs is then clearly $H$.

Summing up, if $H$ is the (second) intersection of the circumference through $A, B$ and $\mathrm{S}$ with the circumference with centre $K$ and radius $\overline{K A}$, we have (see Fig. 24):

$$
\begin{aligned}
\overline{B R} & =\overline{R S}=\overline{R A}=\overline{R H} \\
\text { Hypothesis : } \overline{T A}=\overline{T S} & \text { Thesis : } \overline{H J}=\overline{J B} \\
\overline{K A} & =\overline{K H}
\end{aligned}
$$

Equality of the triangles RKA and RKH implies that $K \hat{H} R=K \hat{A} R$, which added to $R \hat{H} B=R \hat{B} H$ yields

$$
K \hat{H} B=K \hat{A} R+R \hat{B} H
$$

Formula (1) implies that triangles $B O F$ and $F A R$ are similar, and so that $J \hat{B} R=K \hat{A} R$; by substitution of this equality in (3) we obtain that $K \hat{H} B=J \hat{B} R+R \hat{B} H=J \hat{B} H$, thus the thesis.

With regard to the sufficient condition it is enough to choose a point $H$ on $\operatorname{arc} A S$ and determine the intersection $K$ of the axis of the segment $H A$ with $O A$; the circumference with centre $\mathrm{K}$ and radius $\overline{K H}$ will then pass through $A$ and have $A T$ as tangent; once that I show that $\mathrm{K}$ exists and is such that

$$
\frac{\overline{A O}-\overline{O B}}{1-\cos \alpha}<\overline{O K}<\overline{O A}
$$

naming $\mathrm{J}$ the intersection of $K H$ with $O A$, the proof that $H$ is the connection point proceeds formally as the second part (2) of the proof of the necessary condition.

In order to prove (4) I will call $\mathrm{V}$ the intersection of the parallel to $O B$ through $\mathrm{S}$ with OA; I start by pointing out that the equality of triangles $A V R$ and $V R S$ 


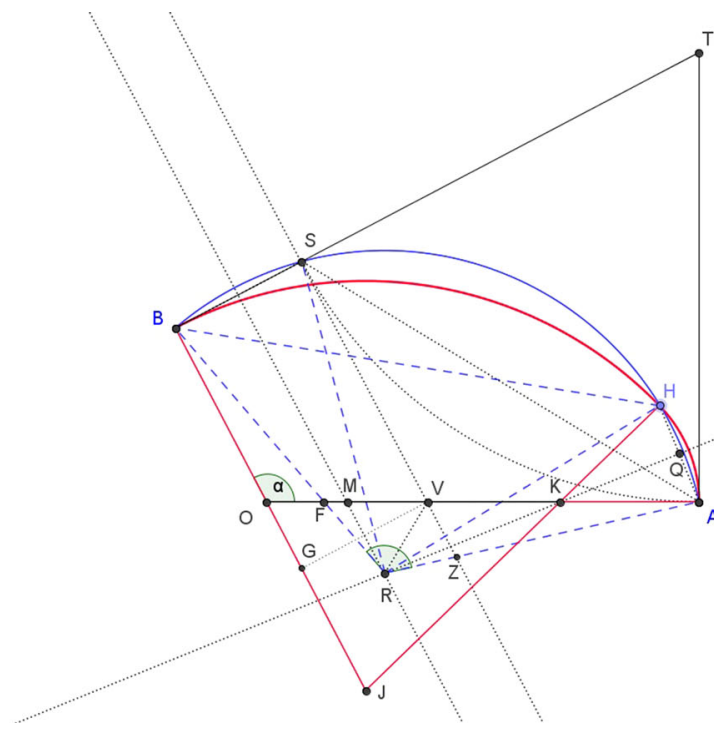

Fig. 24 The construction needed to prove Theorem 1

$(\overline{A V}=\overline{V S}$ because right triangles $A V T$ and $V S T$ are equal) implies that $V R$ divides $Z \hat{V} O$ in two equal parts, and so

$$
O \hat{V} R=R \hat{V} Z=\frac{\alpha}{2}
$$

moreover, if $\mathrm{G}$ is the foot of the perpendicular from $V$ onto $B O$, we have

$$
\overline{O V}=\overline{A O}-\overline{A V}=\overline{A O}-\overline{V S}=\overline{A O}-\overline{B G}=\overline{A O}-(\overline{B O}+\overline{O V} \cdot \cos (\pi-\alpha))
$$

and first and last terms yield $\overline{O V}=\frac{\overline{A O}-\overline{B O}}{1+\cos (\pi-\alpha)}=\frac{\overline{A O}-\overline{B O}}{1-\cos \alpha}$.

I now only need to show that $\overline{O V}<\overline{O K}<\overline{O A}$. Point $R$ is on the axis of $B S$, thus inside the strip determined by $B O$ and $S V$. If it were on the same side as $\mathrm{H}$ w.r.t. OA, we would have a non-tangled quadrilateral $A O B R$ having the angle in $O$ measuring $\alpha$ and the one in $R$ measuring $2 \pi-\alpha$-the concave version of $A \hat{R} B=\alpha$ (notice that (1) has been proved independently of $\mathrm{K}$ )—which is unacceptable. This implies, since $R$ belongs to $K Q$ (see Fig. 24), that point $K$ exists and lies between $A$ and $M$ (intersection of the axis of the segment $B S$ with $O A$ ), and so $\overline{O K}<\overline{O A}$. On the other hand $K \equiv V$ is no possible option, otherwise, since $\overline{A V}=\overline{V S}$, we would have $\overline{A K}=$ $\overline{K S}$ and the circumference with centre $\mathrm{K}$ would have $\mathrm{S}$ in common with the circumference through $A, B$ and $\mathrm{S}$, i.e. $H \equiv S$, forbidden by our hypotheses. Finally, it is also impossible that $\overline{O M}<\overline{O K}<\overline{O V}$, otherwise $O \hat{K} R$ would be an external angle of the triangle $K V R$, and for a known theorem it would be $O \hat{K} R>O \hat{V} R$; this would imply, being $A \tilde{K} Q=O \hat{K} R$, that $A \hat{K} Q>O \hat{V} R$, and thus-by observation of the triangle $Q A K$ - that 


$$
Q \hat{A} K=\frac{\pi}{2}-A \hat{K} Q<\frac{\pi}{2}-O \hat{V} R
$$

But $S \hat{A} K$ is a part of $Q \hat{A} K$, and so $Q \hat{A} K>S \hat{A} K=\frac{\pi-\alpha}{2}$ (see triangle $S A V$ ), comparing which with (6) we obtain $\frac{\pi}{2}-O \hat{V} R>\frac{\pi-\alpha}{2}$, implying $O \hat{V} R<\frac{\alpha}{2}$, in contrast with (5).

Proof of Theorem 4 The construction preceding Theorem 1 can be repeated, although $F$ in Fig. 7 needs to be chosen on the same side of $B$ w.r.t. $O$. The remark following it yields the slightly different condition for $K$ :

$$
\overline{O K}>\frac{\overline{O A}+\overline{O B}}{1-\cos \alpha}
$$

Looking now inside Theorem 1 itself we notice that the proof regarding angle $B \hat{R} A$ does not need any changes. The necessary condition is also applicable to our new case, given that $K$ satisfies condition (7). I will though re-write the proof of the sufficient condition due to the few changes needed.

With regard to the sufficient condition it is enough to choose a point $H$ on $\operatorname{arc} A S$ and determine the intersection $K$ of the axis of the segment $H A$ with $O A$; the circumference with centre $\mathrm{K}$ and radius $\overline{K H}$ will then pass through $A$ and have $A T$ as tangent; once that $\mathrm{I}$ show that $\mathrm{K}$ exists and is such that

$$
\frac{\overline{A O}+\overline{O B}}{1-\cos \alpha}<\overline{O K}<\overline{O A}
$$

naming $\mathrm{J}$ the intersection of $K H$ with $O A$, the proof that $H$ is the connection point proceeds formally as the second part (2) of the proof of the necessary condition in Theorem 1.

In order to prove (8) I will call $\mathrm{V}$ the intersection of the parallel to $O B$ through $\mathrm{S}$ with OA; I start by pointing out that the equality of triangles $A V R$ and $V R S$ $(\overline{A V}=\overline{V S}$ because right triangles $A V T$ and $V S T$ are equal) implies that $V R$ divides $Z \hat{V} O$ in two equal parts, and so

$$
O \hat{V} R=R \hat{V} Z=\frac{\alpha}{2}
$$

moreover, if $\mathrm{G}$ is the foot of the perpendicular from $V$ onto $B O$, we have

$$
\overline{O V}=\overline{A O}-\overline{A V}=\overline{A O}-\overline{V S}=\overline{A O}-\overline{B G}=\overline{A O}-(\overline{O V} \cdot \cos (\pi-\alpha)-\overline{B O})
$$

and first and last terms yield $\overline{O V}=\frac{\overline{A O}+\overline{B O}}{1+\cos (\pi-\alpha)}=\frac{\overline{A O}+\overline{B O}}{1-\cos \alpha}$.

I now only need to show that $\overline{O V}<\overline{O K}<\overline{O A}$. Point $R$ is on the axis of $B S$, thus inside the strip determined by $B O$ and $S V$. If it were on the same side as $\mathrm{H}$ w.r.t. OA, we would have a tangled quadrilateral $R Z V F$ having the angle in $V$ measuring $\alpha$ and the one in $R$ measuring $2 \pi-\alpha$-the concave version of $A \hat{R} B=\alpha$-which is unacceptable also for tangled quadrilaterals (the sum of the internal angles has to be smaller than $2 \pi$ since this measure is reached adding the two opposite angles at the 
knot). This implies, since $R$ belongs to $K Q$ (see Fig. 25), that point $K$ exists and lies between $A$ and $M$ (intersection of the axis of the segment $B S$ with $O A$ ), and so $\overline{O K}<\overline{O A}$. On the other hand $K \equiv V$ is no possible option, otherwise, since $\overline{A V}=\overline{V S}$, we would have $\overline{A K}=\overline{K S}$ and the circumference with centre $\mathrm{K}$ would have $\mathrm{S}$ in common with the circumference through $A, B$ and $\mathrm{S}$, i.e. $H \equiv S$, forbidden by our hypotheses. Finally, it is also impossible that $\overline{O M}<\overline{O K}<\overline{O V}$, otherwise $O \hat{K} R$ would be an external angle of the triangle $K V R$, and for a known theorem it would be $O \hat{K} R>O \hat{V} R$; this would imply, being $A \tilde{K} Q=O \hat{K} R$, that $A \hat{K} Q>O \hat{V} R$, and thus - by observation of the triangle $Q A K$ - that

$$
Q \hat{A} K=\frac{\pi}{2}-A \hat{K} Q<\frac{\pi}{2}-O \hat{V} R
$$

But $S \hat{A} K$ is a part of $Q \hat{A} K$, and so $Q \hat{A} K>S \hat{A} K=\frac{\pi-\alpha}{2}$ (see triangle $S A V$ ), comparing which with (10) we obtain $\frac{\pi}{2}-O \hat{V} R>\frac{\pi-\alpha}{2}$, implying $O \hat{V} R<\frac{\alpha}{2}$, in contrast with (9).

Proof of Theorem 5 In this first part I will assume that $H \in \Gamma_{1}-\left\{A, B, S_{1}, S_{2}\right\}$.

Consider $H$ on arc $B S_{1}$ (Fig. 26). Call $J$ the intersection between the axis of $H B$ and line $O B$, existing because $H B$ is not orthogonal to $O B$, which were the case only if $H \equiv S_{1}$, and then $K$ the intersection between the axis of $H A$ and line $O A$, existing because $H A$ is not orthogonal to $O A$, which were the case only if $H \equiv S_{2}$. Let $\Gamma_{3}$ be the circumference with centre $K$ and radius $\overline{A K}$, tangent in $A$ to $t_{A}$, and $\Gamma_{4}$ the one with centre $J$ and radius $\overline{J B}$ tangent in $B$ to $t_{B}$. They obviously meet in $H$, so all we need to prove is that $H, J$ and $K$ are co-linear, to prove tangency in $H$. Figure 26 shows that

$$
K \hat{H} J=J \hat{H} R+R \hat{H} K=J \hat{B} R+R \hat{A} K=(\pi-R \hat{B} O)+R \hat{A} K=\pi,
$$

the last equality descending from the fact that $O A$ and $R B$ form similar trianglessee properties of the system of tangents.

Consider $H$ on $\operatorname{arc} B S_{2}$. Find $J, K, \Gamma_{3}$ and $\Gamma_{4}$ as above. This time I will prove that $R \hat{H} J=R \hat{H} K$. From Fig. 27 we get that $R \hat{H} J-R \hat{H} K=R \hat{B} J-R \hat{A} K=0$, again from the fact that $O A$ and $R B$ form with $\overline{O B}$ and $\overline{A R}$ similar triangles.

Consider $H$ on $\operatorname{arc} A S_{2}$. Find $J, K, \Gamma_{3}$ and $\Gamma_{4}$ as above. From Fig. 28 we get that $K \hat{H} J=J \hat{H} R+R \hat{H} K=J \hat{B} R+R \hat{A} K=J \hat{B} R+(\pi-R \hat{A} O)=\pi$, with the same argument as before. Finally consider $H$ on arc $A S_{1}$. From Fig. 29 we have $R \hat{H} J-R \hat{H} K=R \hat{B} J-R \hat{A} K=0$, again using the same argument.

The above proof procedure works also in a very similar way if the sequence of points on $\Gamma_{1}$ is different and/or if $O A T B$ is tangled or becomes a triangle.

For the second part I will assume that $H \in \Gamma_{2}-\left\{A, B, S_{3}, S_{4}\right\}$. Consider $H$ on arc $B S_{3}$ (see Fig. 30). Call $J$ the intersection between the axis of $H B$ and line $O B$, existing because $H B$ is not orthogonal to $O B$, which were the case only if $H \equiv S_{4}$, and then $K$ the intersection between the axis of $H A$ and line $O A$, existing because $H A$ is not orthogonal to $O A$, which were the case only if $H \equiv S_{3}$. Let $\Gamma_{3}$ be the circumference with centre $K$ and radius $\overline{A K}$, tangent in $A$ to $t_{A}$, and $\Gamma_{4}$ the one with centre $J$ and radius $\overline{J B}$ tangent in $B$ to $t_{B}$. They obviously meet in $H$, so all we need 


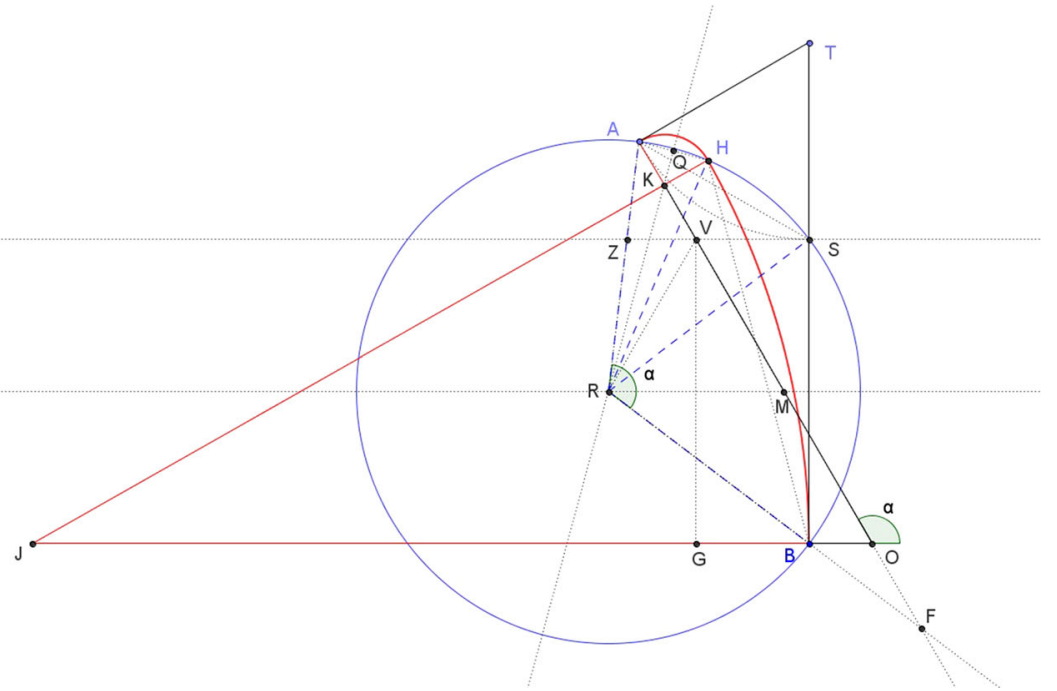

Fig. 25 Proving that the CL works also when the quadrilateral is tangled

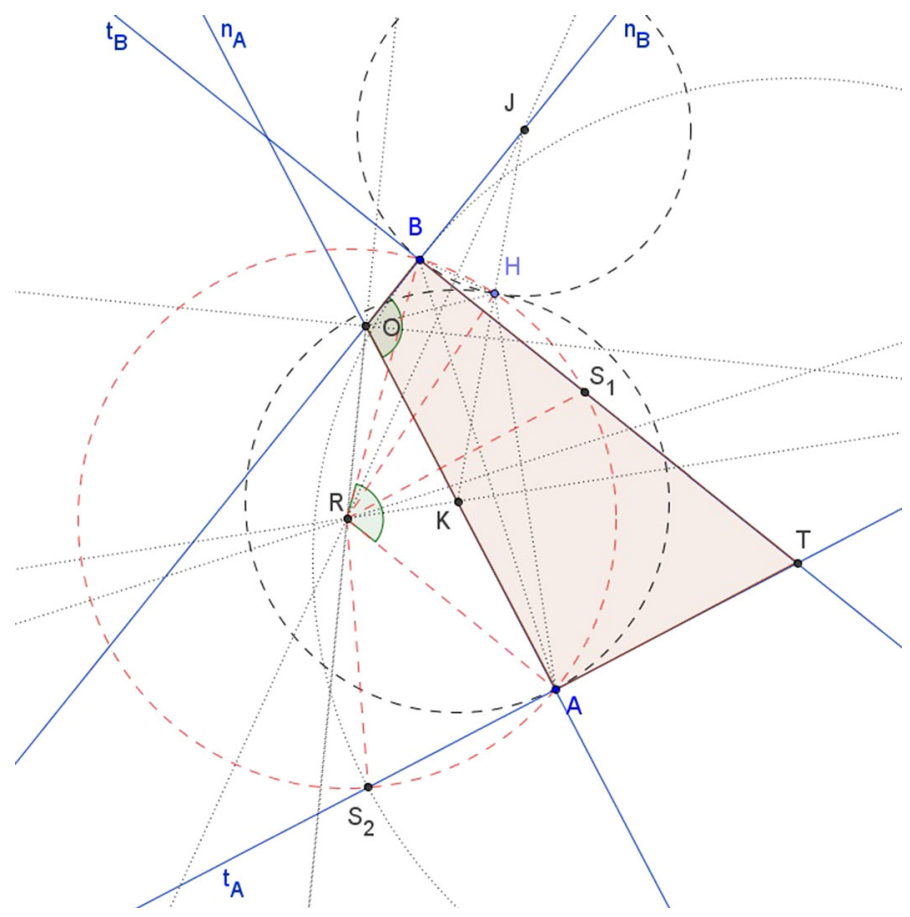

Fig. 26 Proving the first part of Theorem 5 when $H$ is on $\operatorname{arc} B S_{1}$ 


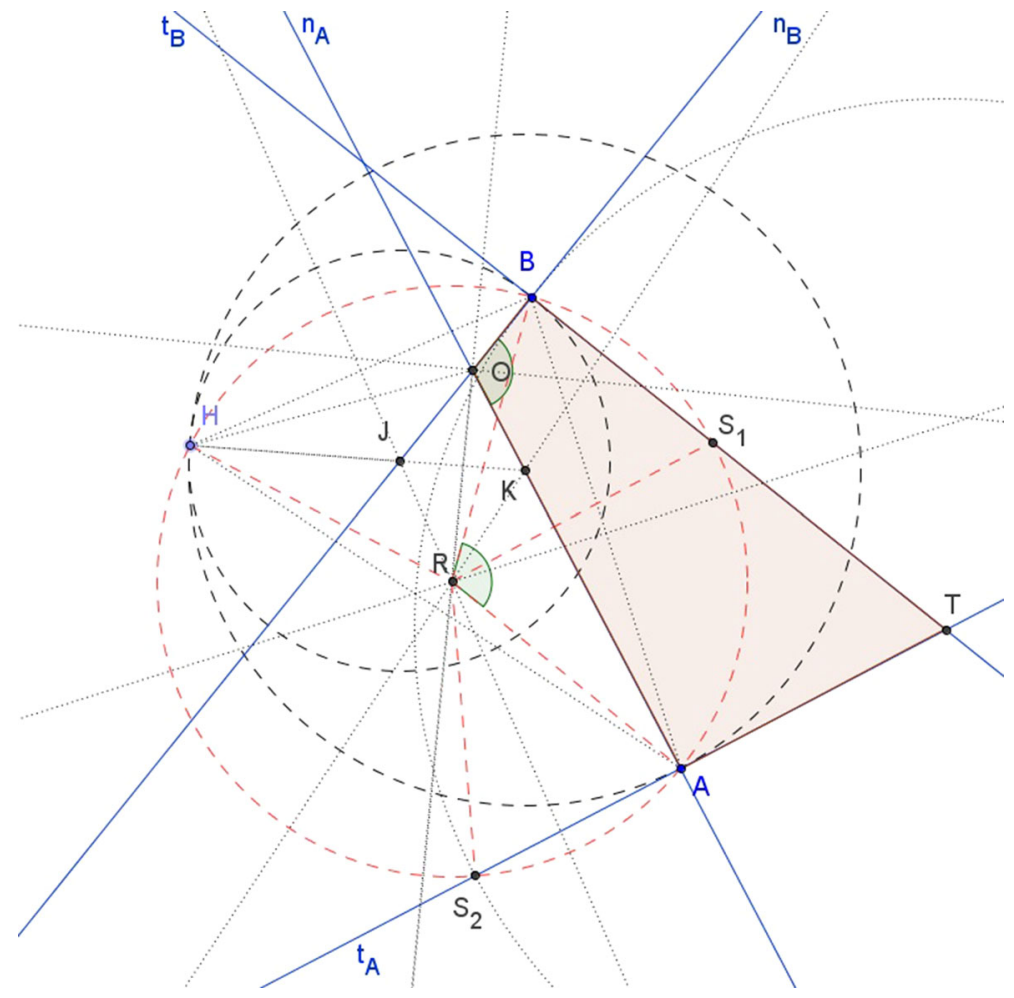

Fig. 27 Proving the first part of Theorem 5 when $H$ is on $\operatorname{arc} B S_{2}$

to prove is that $H, J$ and $K$ are co-linear, to prove tangency in $H$. Figure 30 shows that $F \hat{H} J-F \hat{H} K=F \hat{B} J-F \hat{A} K=(\pi-F \hat{B} O)-F \hat{A} O$; remembering now that $A \hat{F} B+A \hat{O} B=\beta+\alpha=\pi, \quad$ we $\quad$ get $\quad(\pi-F \hat{B} O)-F \hat{A} O=\pi-(2 \pi-(A \hat{F} B+$ $A \hat{O} B))=0$, and so $F, H$ and $J$ are co-linear.

Choose $H$ on arc $S_{4} S_{3}$. By looking at Fig. 31 using again the properties of the angles in $A F B O$ we see that $J \hat{H} K=J \hat{H} F+F \hat{H} K=(\pi-F \hat{B} O)+(\pi-F \hat{A} O)=$ $2 \pi-(\alpha+\beta)=\pi$ and so $F, H$ and $J$ are co-linear.

Choose $H$ on arc $S_{4} A$ (see Fig. 32). We have, using the same arguments as above, $F \hat{H} J-F \hat{H} K=F \hat{B} O-F \hat{A} K=F \hat{B} O-(\pi-F \hat{A} O)=\pi-\pi=0$.

Finally, with $H$ on arc $A B$ (Fig. 33), we have $J \hat{H} K=J \hat{H} F+F \hat{H} K=$ $O \hat{A} F+F \hat{B} O=\pi$, as usual. The above proof procedure works also in a very similar way if the sequence of points on $\Gamma_{2}$ is different and/or if OATB is tangled or becomes a triangle.

The proof is now complete.

Proof of Lemma 6 Any circumference $\Gamma_{3}$ through $A$ tangent to $t_{A}$ has its centre on $n_{A}$ (see Fig. 34). Let $H$ be the tangency point with $\Gamma_{4}$. By means of Archimedes's Lemma we know that the two other intersections of any line through $H$ with $\Gamma_{3}$ and 


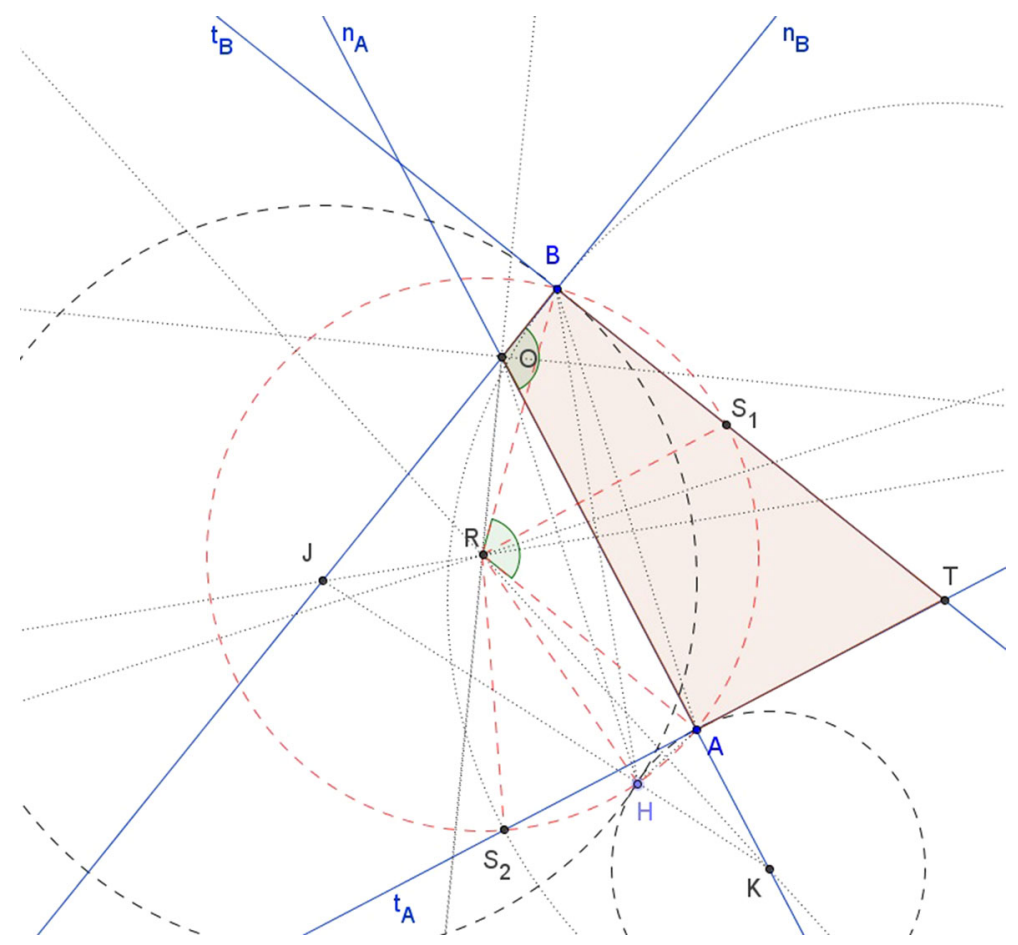

Fig. 28 Proving the first part of Theorem 5 when $H$ is on $\operatorname{arc} A S_{2}$

$\Gamma_{4}$ form parallel lines when joined to the corresponding centre, which means that $F J$ is parallel to $n_{A}$. This means that the described construction yields all possible circumferences tangent in $A$ to $t_{A}$ and to the circumference $\Gamma_{4}$.

Proof of Theorem 7 We draw our usual system of tangents with base points $A$ and $B$ as well as the centres $R$ and $F$ of the circumferences $\Gamma_{1}$ and $\Gamma_{2}$ of the supposed CL. I have to construct all possible pairs of circumferences $\Gamma_{3}$ and $\Gamma_{4}$ and show that their connection point $H$ belongs either to $\Gamma_{1}$ or to $\Gamma_{2}$. To do so I will calculate the angles formed by each one with $A$ and $B$. Having set as usual $A \hat{R} B=A \hat{O} B=\alpha$ and thus $A \hat{F} B=A \hat{T} B=\pi-\alpha$. I will prove that each connection point

- when on $R$ 's side w.r.t. $O A$ forms with $A$ and $B$ either an angle of $\frac{\alpha}{2}$ thus belonging to $\Gamma_{1}$ or an angle of $\pi-\frac{\pi-\alpha}{2}=\frac{\pi+\alpha}{2}$ thus belonging to $\Gamma_{2}$, or

- when on $F$ 's side w.r.t. $O A$ forms with $A$ and $B$ either an angle of $\pi-\frac{\alpha}{2}$ thus belonging to $\Gamma_{1}$ or an angle of $\frac{\pi-\alpha}{2}$ thus belonging to $\Gamma_{2}$.

I need to examine all cases according to the possible positions of the centre $J$ of $\Gamma_{4}$ along $O B$. Each one yields a pair of feasible $\Gamma_{3}$ circumferences.

The proofs for the cases of $O A T B$ becoming a triangle or a tangled quadrilateral represent a mere repetition of the techniques used here. 


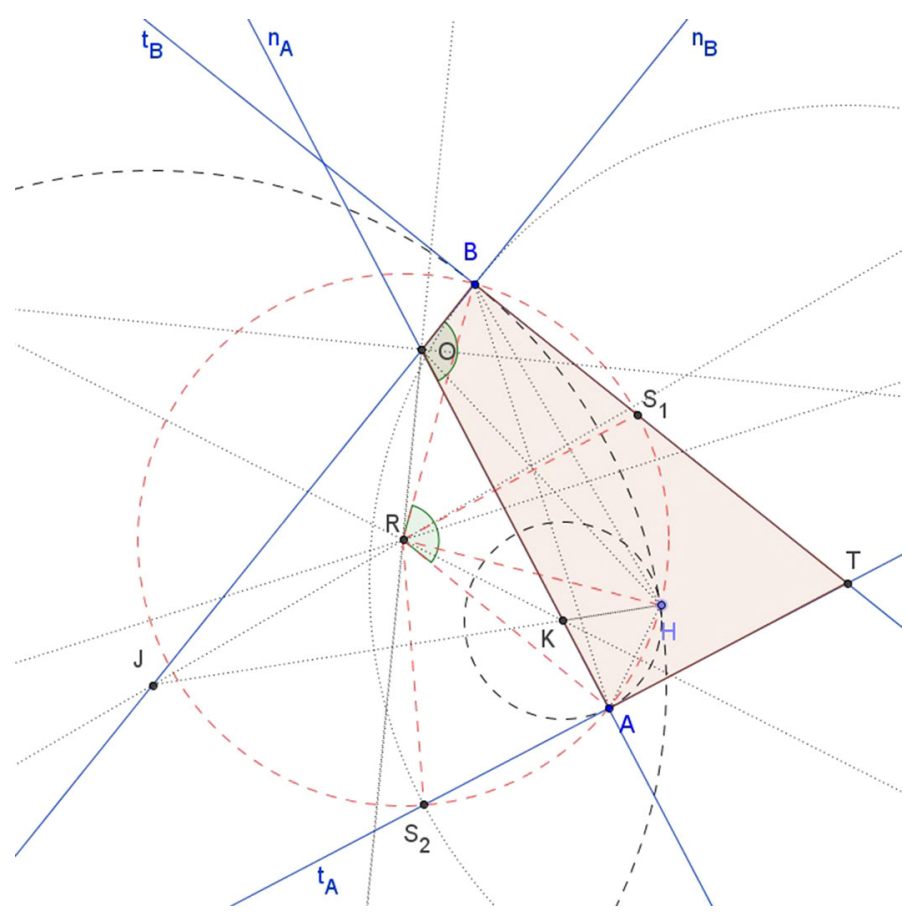

Fig. 29 Proving the first part of Theorem 5 when $H$ is on $\operatorname{arc} A S_{1}$

Case $1-$ When $J$ is opposite to $B$ w.r.t. $O$ and $J$ is far enough from $B$ for $\Gamma_{4}$ to include $A$ the two connecting points $H_{1}$ and $H_{2}$, found with the method illustrated before Lemma 6 , lie beyond $B A$ - as can be easily proved-and the two (only) possible circumferences tangent to $\Gamma_{3}$ are those shown in Fig. 35 with centres $K_{1}$ and $K_{2}$. We first of all see that

$$
A \hat{H}_{2} B=A \hat{H}_{2} J+J \hat{H}_{2} B=O \hat{A} H_{2}+O \hat{B} H_{2}
$$

the last inequality since $\overline{A K_{2}}=\overline{K_{2} H_{2}}$ and $\overline{J H_{2}}=\overline{J B}$. Then we use the fact that $O \hat{A} H_{2}=H_{2} \hat{A} B+B \hat{A} O$ and that $O \hat{B} H_{2}=A \hat{B} H_{2}+A \hat{B} O$ and we include these two in the previous relation:

$$
\begin{aligned}
A \hat{H}_{2} B & =H_{2} \hat{A} B+B \hat{A} O+A \hat{B} H_{2}+A \hat{B} O=\left(H_{2} \hat{A} B+A \hat{B} H_{2}\right)+(B \hat{A} O+A \hat{B} O) \\
& =\left(\pi-A \hat{H}_{2} B\right)+(\pi-\alpha),
\end{aligned}
$$

the last inequality observing triangles $H_{2} A B$ and $O A B$. Using the first and last term to calculate $A \hat{H}_{2} B$ we get $A \hat{H}_{2} B=\pi-\frac{\alpha}{2}$, which proves that $H_{2} \in \Gamma_{1}$. Now for $H_{1}$ :

$$
A \hat{H}_{1} B=J \hat{H}_{1} B-A \hat{H}_{1} J=O \hat{B} H_{1}-\left(\pi-O \hat{A} H_{1}\right),
$$

the last inequality since $\overline{A H_{1}}=\overline{K_{1} H_{1}}$ and $\overline{J H_{1}}=\overline{J B}$. Then we use $O \hat{B} H_{1}=$ $O \hat{B} A+A \hat{B} H_{1}$ and $O \hat{A} H_{1}=H_{1} \hat{A} B+B \hat{A} O$ and this yields: 




Fig. 30 Proving the second part of Theorem 5 when $H$ is on arc $B S_{3}$

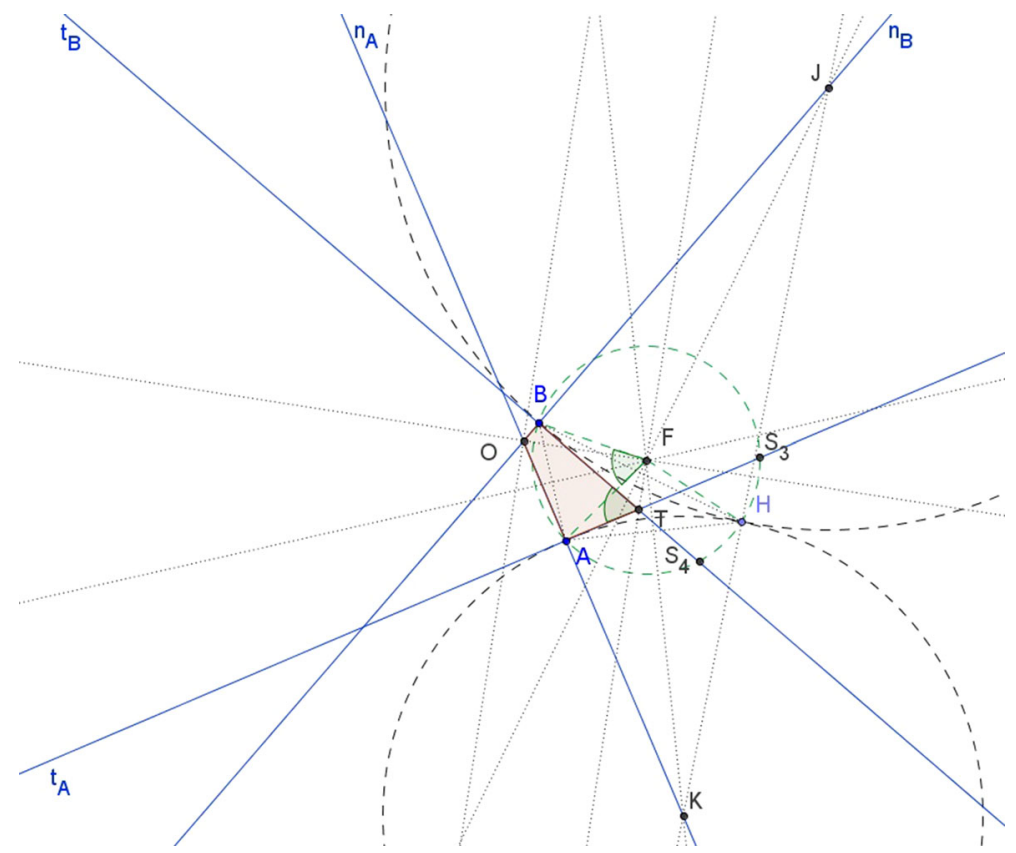

Fig. 31 Proving the second part of Theorem 5 when $H$ is on $\operatorname{arc} S_{4} S_{3}$ 


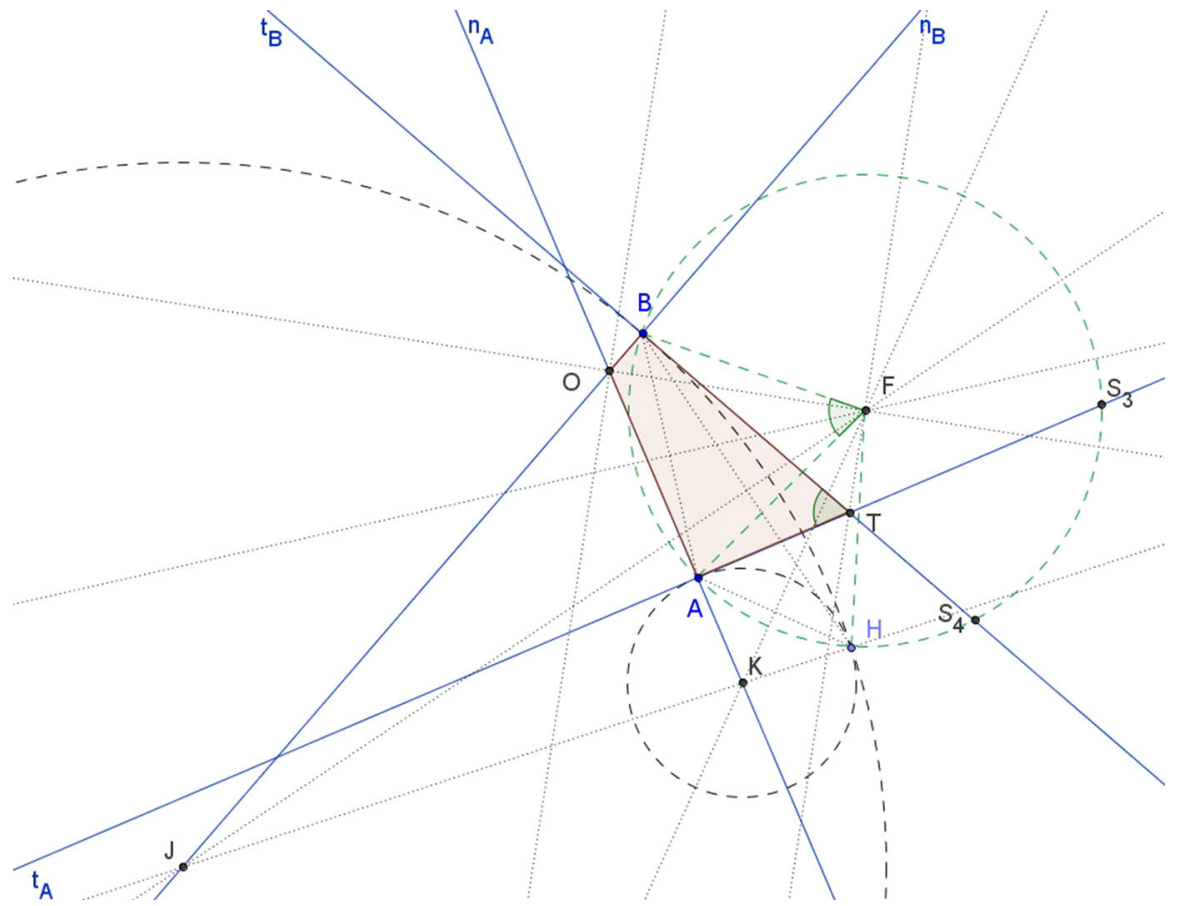

Fig. 32 Proving the second part of Theorem 5 when $H$ is on $\operatorname{arc} S_{4} A$



Fig. 33 Proving the second part of Theorem 5 when $H$ is on $\operatorname{arc} B A$ 


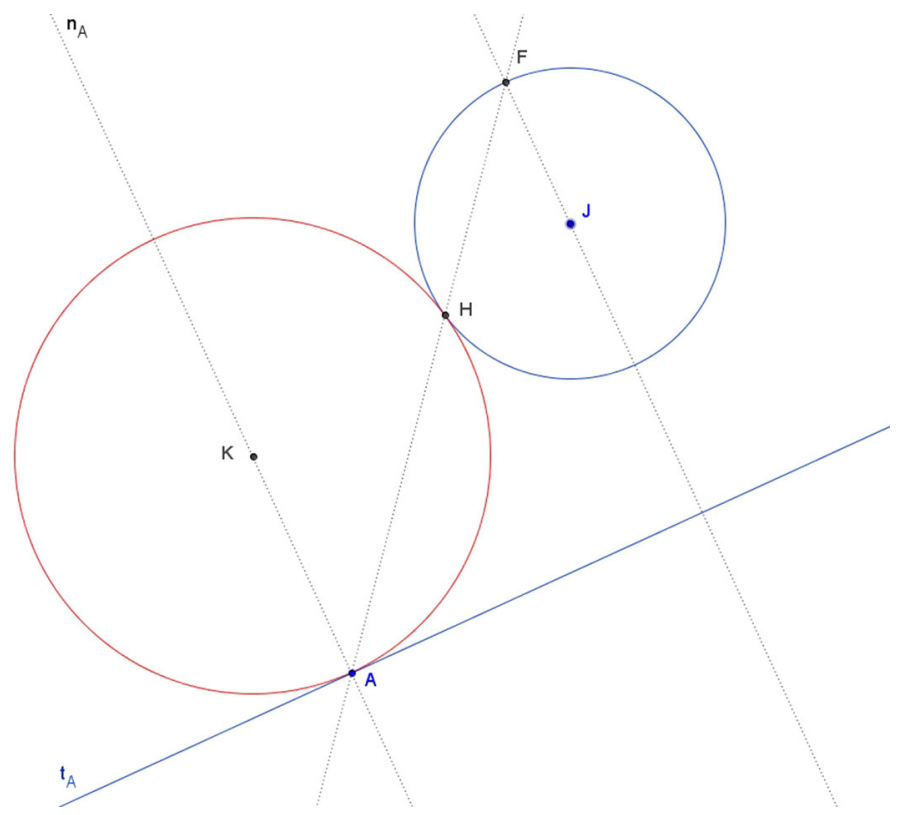

Fig. 34 Proving Lemma 6

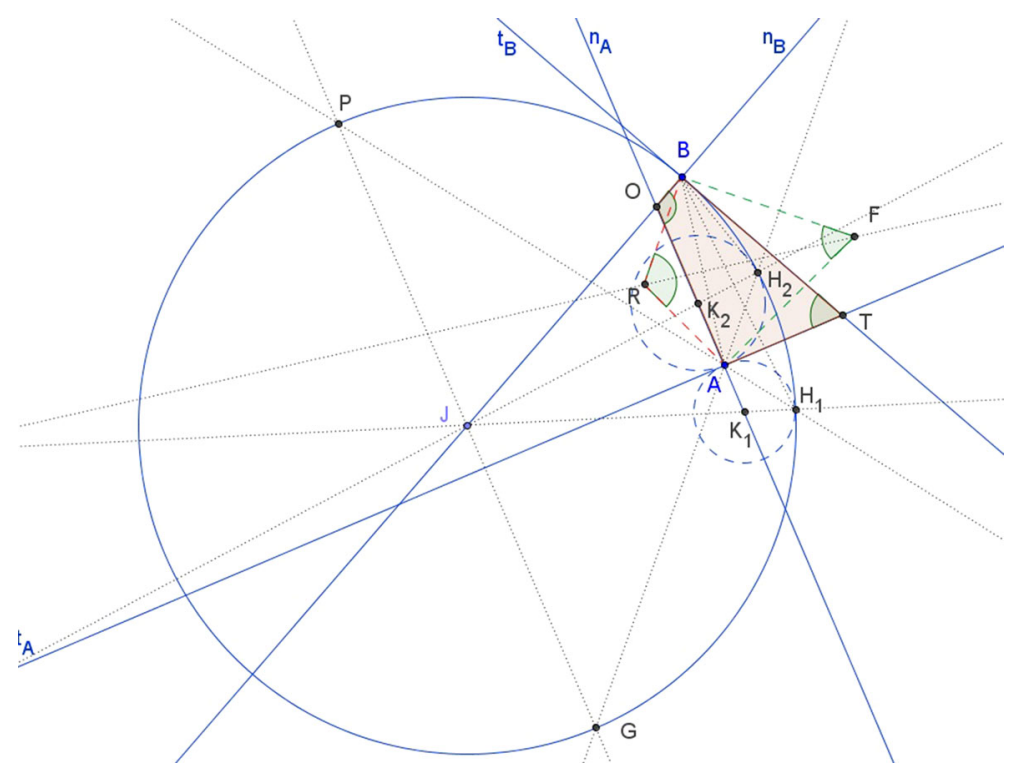

Fig. 35 Proving Theorem 7-Case 1 


$$
\begin{aligned}
A \hat{H}_{1} B & =O \hat{B} A+A \hat{B} H_{1}-\left(\pi-\left(H_{1} \hat{A} B+B \hat{A} O\right)\right) \\
& =(O \hat{B} A+B \hat{A} O)+\left(A \hat{B} H_{1}+H_{1} \hat{A} B\right)-\pi=(\pi-\alpha)+\left(\pi-A \hat{H}_{1} B\right)-\pi
\end{aligned}
$$

considering triangles $H_{1} A B$ and $O A B$. Using the first and last term to calculate $A \hat{H}_{1} B$ we get $A \hat{H}_{1} B=\frac{\pi-\alpha}{2}$, which proves that $H_{1} \in \Gamma_{2}$.

Case 2-For positions of $J$ closer to $O$, as long as $H_{2}$ is on the same side as $A$ w.r.t. $J B$, we can refer to both situations illustrated in Fig. 36; when $\Gamma_{4}$ is tangent to $T A$ there is only one circumference tangent to $\Gamma_{3}$. Points $H_{1}$ and $H_{2}$ are still on $R$ 's side w.r.t. $O A$. We have that:

$$
A \hat{H}_{2} B=A \hat{H}_{2} J-J \hat{H}_{2} B=O \hat{A} H_{2}-O \hat{B} H_{2},
$$

since $\overline{A K_{2}}=\overline{K_{2} H_{2}}$ and $\overline{J H_{2}}=\overline{J B}$. Then we use $O \hat{A} H_{2}=H_{2} \hat{A} B-B \hat{A} O$ and $O \hat{B} H_{2}=A \hat{B} O-A \hat{B} H_{2}$ and we get:

$$
\begin{aligned}
A \hat{H}_{2} B & =H_{2} \hat{A} B-B \hat{A} O-\left(A \hat{B} O-A \hat{B} H_{2}\right)=\left(H_{2} \hat{A} B+A \hat{B} H_{2}\right)-(B \hat{A} O+A \hat{B} O) \\
& =\left(\pi-A \hat{H}_{2} B\right)-(\pi-\alpha)
\end{aligned}
$$

considering $\mathrm{H}_{2} A B$ and $O A B$. Using the first and last term we get $A \hat{H}_{2} B=\frac{\alpha}{2}$, proving that $H_{2} \in \Gamma_{1}$. Now for $H_{1}$ :

$$
A \hat{H}_{1} B=\operatorname{concave}\left(A \hat{H}_{1} J\right)-J \hat{H}_{1} B=\left(\pi+O \hat{A} H_{1}\right)-O \hat{B} H_{1},
$$

since $\overline{A H_{1}}=\overline{K_{1} H_{1}}$ and $\overline{J H_{1}}=\overline{J B}$. Then we use $O \hat{B} H_{1}=O \hat{B} A-A \hat{B} H_{1}$ and $O \hat{A} H_{1}=H_{1} \hat{A} B-B \hat{A} O$ and so:

$$
\begin{aligned}
A \hat{H}_{1} B & =\left(\pi+\left(H_{1} \hat{A} B-B \hat{A} O\right)\right)-\left(O \hat{B} A-A \hat{B} H_{1}\right) \\
& =\pi-(O \hat{B} A+B \hat{A} O)+\left(A \hat{B} H_{1}+H_{1} \hat{A} B\right)=\pi-(\pi-\alpha)+\left(\pi-A \hat{H}_{1} B\right),
\end{aligned}
$$

considering triangles $H_{1} A B$ and $O A B$. Using the first and last term we get $A \hat{H}_{1} B=$ $\frac{\pi+\alpha}{2}$, which proves that $H_{1} \in \Gamma_{2}$.

Case 3-When point $H_{2}$ is on $J B$ we have (Fig. 37):

$$
A \hat{H}_{2} B=A \hat{H}_{2} J=O \hat{A} H_{2},
$$

since $\overline{A K_{2}}=\overline{K_{2} H_{2}}$. Then we use $O \hat{A} H_{2}=H_{2} \hat{A} B-B \hat{A} O$ and we get:

$$
\begin{aligned}
A \hat{H}_{2} B & =H_{2} \hat{A} B-B \hat{A} O=\left(\pi-\left(A \hat{H}_{2} B+O \hat{B} A\right)\right)-B \hat{A} O \\
& =\pi-(B \hat{A} O+O \hat{B} A)-A \hat{H}_{2} B=\alpha-A \hat{H}_{2} B,
\end{aligned}
$$

considering triangles $H_{2} A B$ and $O A B$. Using the first and last term we get $A \hat{H}_{2} B=$ $\frac{\alpha}{2}$, which proves that $H_{2} \in \Gamma_{1}$. For $H_{1}$ the proof is identical to that of Case 2 .

Case 4-For positions of $J$ even closer to $O$, when $H_{2}$ is on the opposite side of $A$ w.r.t. $J B$, we can refer to Fig. 38. Points $H_{1}$ and $H_{2}$ are still on $R$ 's side w.r.t. $O A$. We have that: 


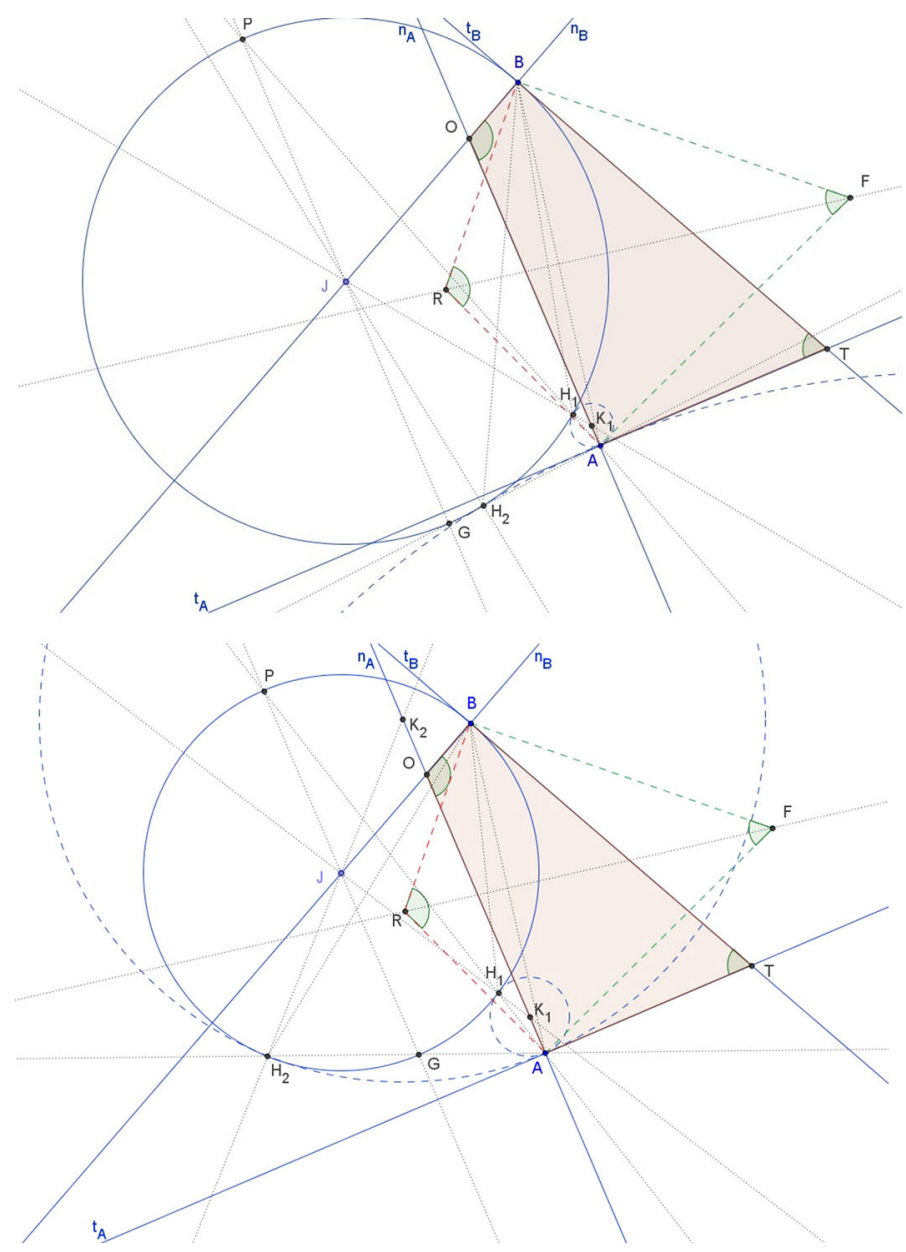

Fig. 36 Proving Theorem 7-Case 2

$$
A \hat{H}_{2} B=A \hat{H}_{2} J+J \hat{H}_{2} B=O \hat{A} H_{2}+O \hat{B} H_{2},
$$

since $\overline{A K_{2}}=\overline{K_{2} H_{2}}$ and $\overline{J H_{2}}=\overline{J B}$. Then since $O \hat{A} H_{2}=H_{2} \hat{A} B-B \hat{A} O$ and $O \hat{B} H_{2}=$ $A \hat{B} H_{2}-A \hat{B} O$ we get:

$$
\begin{aligned}
A \hat{H}_{2} B & =\left(H_{2} \hat{A} B-B \hat{A} O\right)+\left(A \hat{B} H_{2}-A \hat{B} O\right)=\left(H_{2} \hat{A} B+A \hat{B} H_{2}\right)-(B \hat{A} O+A \hat{B} O) \\
& =\left(\pi-A \hat{H}_{2} B\right)-(\pi-\alpha)
\end{aligned}
$$

considering triangles $H_{2} A B$ and $O A B$. Using the first and last term we get $A \hat{H}_{2} B=\frac{\alpha}{2}$, which proves that $H_{2} \in \Gamma_{1}$. The proof for $H_{1}$ works as in the previous Case 2. 


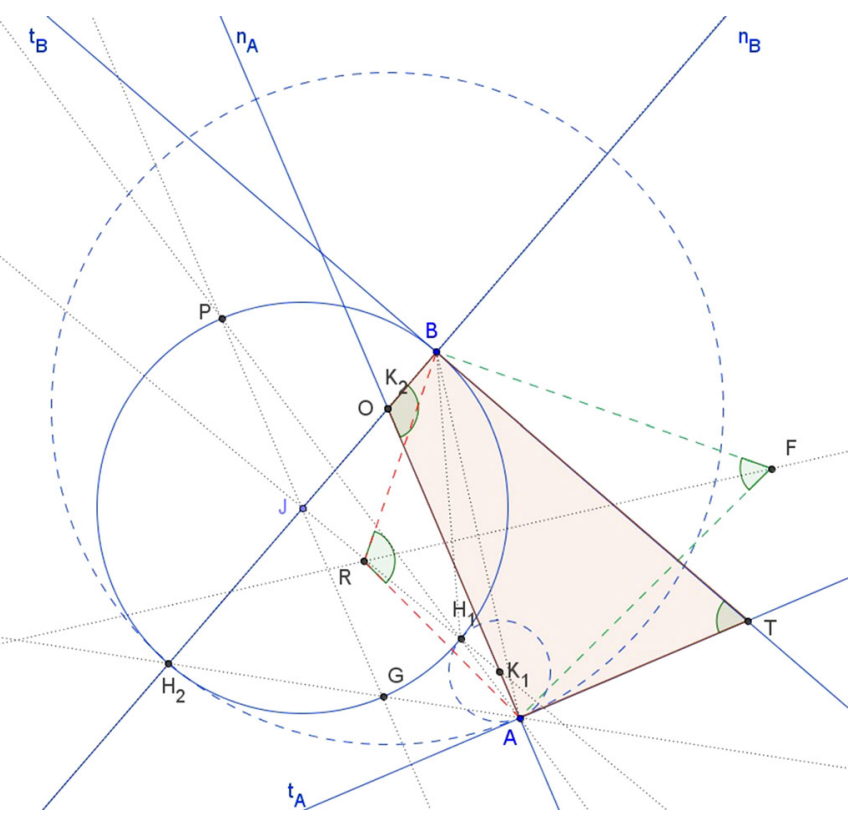

Fig. 37 Proving Theorem 7-Case 3

Case 5-If $J \equiv O$ (see Fig. 39) we have that

$$
A \hat{H}_{2} B=O \hat{B} H_{2}=A \hat{B} H_{2}-A \hat{B} O,
$$

since $\overline{J H_{2}}=\overline{J B}$. And so

$$
\begin{aligned}
A \hat{H}_{2} B & =A \hat{B} H_{2}-A \hat{B} O=\left(\pi-B \hat{A} O-A \hat{H}_{2} B\right)-A \hat{B} O \\
& =\pi-(B \hat{A} O+A \hat{B} O)-A \hat{H}_{2} B=\pi-(\pi-\alpha)-A \hat{H}_{2} B
\end{aligned}
$$

considering triangles $H_{2} A B$ and $O A B$. Using the first and last term we get $A \hat{H}_{2} B=\frac{\alpha}{2}$, which proves that $H_{2} \in \Gamma_{1}$. For $H_{1}$ we have:

$$
A \hat{H}_{1} B=\pi-J \hat{H}_{1} B=\pi-O \hat{B} H_{1},
$$

since $\overline{J H_{1}}=\overline{J B}$. Then we use $O \hat{B} H_{1}=O \hat{B} A-A \hat{B} H_{1}$ and this yields:

$$
\begin{aligned}
A \hat{H}_{1} B & =\pi-\left(O \hat{B} A-A \hat{B} H_{1}\right)=\pi-\left(O \hat{B} A-\left(\pi-A \hat{H}_{1} B-B \hat{A} O\right)\right) \\
& =2 \pi-A \hat{H}_{1} B-(O \hat{B} A+B \hat{A} O)=2 \pi-A \hat{H}_{1} B-(\pi-\alpha),
\end{aligned}
$$

observing triangles $H_{1} A B$ and $O A B$. Using the first and last term we get $A \hat{H}_{1} B=\frac{\pi+\alpha}{2}$, which proves that $H_{1} \in \Gamma_{2}$.

Case 6-Point $J$ lies between $O$ and $B$ and $H_{1}$ and $H_{2}$ are still on $R$ 's side w.r.t. $O A$. We have (Fig. 40)

$$
A \hat{H}_{2} B=J \hat{H}_{2} B-A \hat{H}_{2} J=O \hat{B} H_{2}-O \hat{A} H_{2}
$$




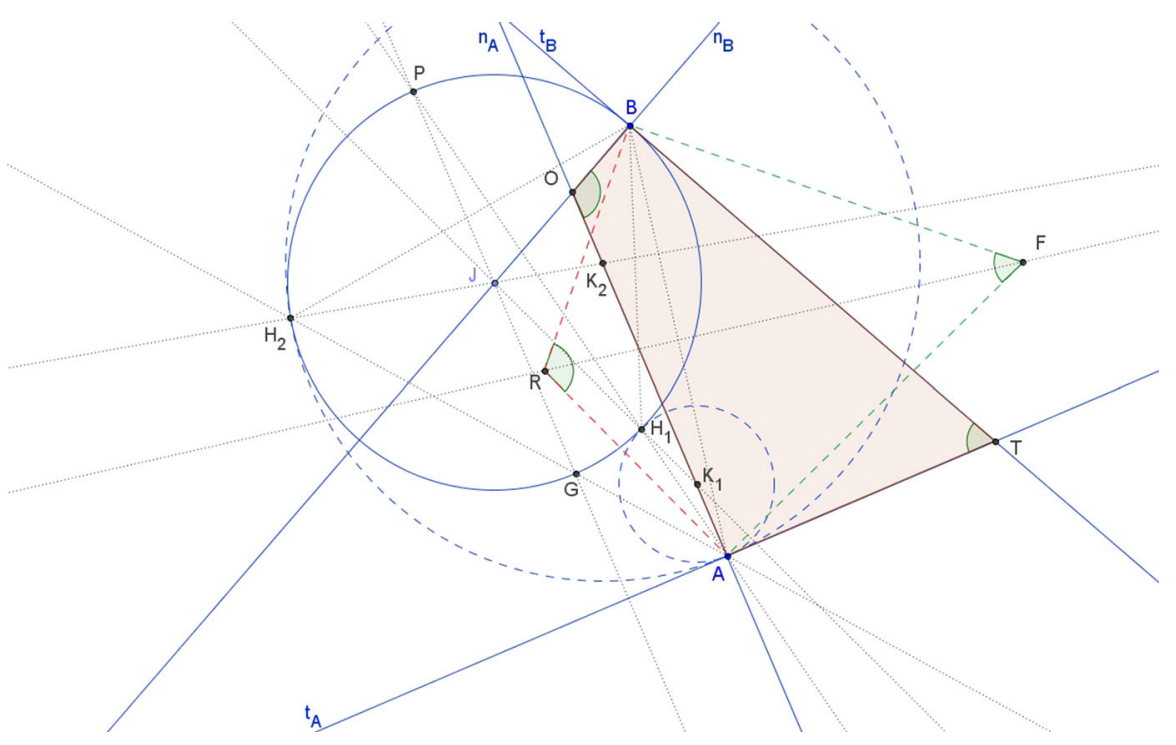

Fig. 38 Proving Theorem 7-Case 4

since $\overline{A K_{2}}=\overline{K_{2} H_{2}}$ and $\overline{J H_{2}}=\overline{J B}$. Then we use $O \hat{A} H_{2}=B \hat{A} O-H_{2} \hat{A} B$ and $O \hat{B} H_{2}=A \hat{B} H_{2}-A \hat{B} O$ and we get:

$$
\begin{aligned}
A \hat{H}_{2} B & =\left(A \hat{B} H_{2}-A \hat{B} O\right)-\left(B \hat{A} O-H_{2} \hat{A} B\right)=\left(H_{2} \hat{A} B+A \hat{B} H_{2}\right)-(B \hat{A} O+A \hat{B} O) \\
& =\left(\pi-A \hat{H}_{2} B\right)-(\pi-\alpha),
\end{aligned}
$$

considering triangles $\mathrm{H}_{2} A B$ and $O A B$. Using the first and last term we get $A \hat{H}_{2} B=\frac{\alpha}{2}$, which proves that $H_{2} \in \Gamma_{1}$.

For $H_{1}$ :

$$
A \hat{H}_{1} B=A \hat{H}_{1} J-J \hat{H}_{1} B=\left(\pi-O \hat{A} H_{1}\right)-O \hat{B} H_{1},
$$

since $\overline{A H_{1}}=\overline{K_{1} H_{1}}$ and $\overline{J H_{1}}=\overline{J B}$. Then we use $O \hat{B} H_{1}=O \hat{B} A-A \hat{B} H_{1}$ and $O \hat{A} H_{1}=B \hat{A} O-H_{1} \hat{A} B$ and this yields:

$$
\begin{aligned}
A \hat{H}_{1} B & =\left(\pi-\left(B \hat{A} O-H_{1} \hat{A} B\right)\right)-\left(O \hat{B} A-A \hat{B} H_{1}\right) \\
& =\pi-(O \hat{B} A+B \hat{A} O)+\left(A \hat{B} H_{1}+H_{1} \hat{A} B\right)=\pi-(\pi-\alpha)+\left(\pi-A \hat{H}_{1} B\right),
\end{aligned}
$$

considering triangles $H_{1} A B$ and $O A B$. Using the first and last term we get $A \hat{H}_{1} B=\frac{\pi+\alpha}{2}$, which proves that $H_{1} \in \Gamma_{2}$.

Case 7-Noting that when $J$ and $B$ coincide no circumference is formed we skip to the case where $J$ is on $B$ 's side w.r.t. $O$ and $H_{2}$ is opposite to $A$ w.r.t. $O B$ (Fig. 41). $H_{1}$ and $H_{2}$ are now on $F$ 's side w.r.t. $O A$. For $H_{2}$ we have: 


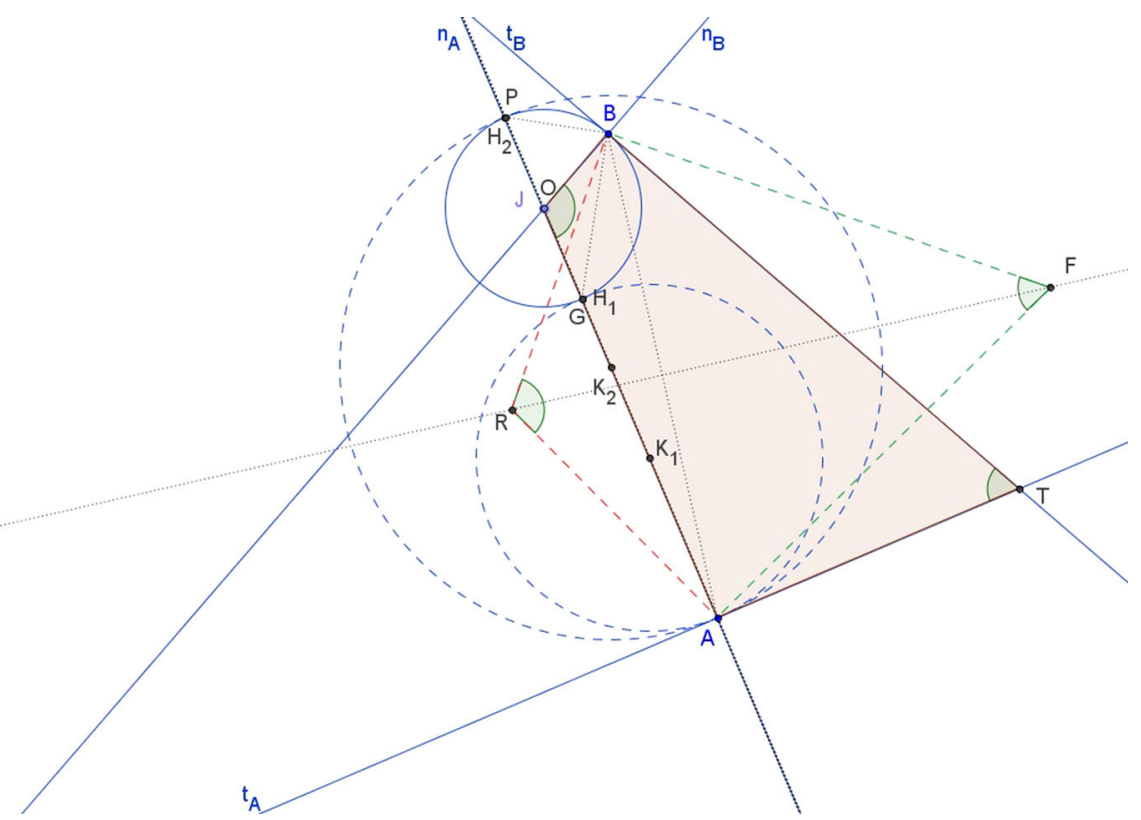

Fig. 39 Proving Theorem 7-Case 5

$$
A \hat{H}_{2} B=J \hat{H}_{2} B+A \hat{H}_{2} J=\pi-O \hat{B} H_{2}+O \hat{A} H_{2},
$$

since $\overline{A K_{2}}=\overline{K_{2} H_{2}}$ and $\overline{J H_{2}}=\overline{J B}$. Then we use $O \hat{A} H_{2}=B \hat{A} O+H_{2} \hat{A} B$ and $O \hat{B} H_{2}=\operatorname{conc}\left(A \hat{B} H_{2}\right)-A \hat{B} O$ :

$$
\begin{aligned}
A \hat{H}_{2} B & =\pi-\left(\operatorname{conc}\left(A \hat{B} H_{2}\right)-A \hat{B} O\right)+\left(B \hat{A} O+H_{2} \hat{A} B\right) \\
& =\pi-\left(\left(2 \pi-A \hat{B} H_{2}\right)-H_{2} A B\right)+(B \hat{A} O+A \hat{B} O) \\
& =\pi-\left(2 \pi-\left(\pi-A \hat{H}_{2} B\right)\right)+(\pi-\alpha)=-A \hat{H}_{2} B+\pi-\alpha,
\end{aligned}
$$

observing triangles $\mathrm{H}_{2} A B$ and $O A B$. Using the first and last term we get $A \hat{H}_{2} B=\frac{\pi-\alpha}{2}$, which proves that $H_{2} \in \Gamma_{2}$.

For $H_{1}$ :

$$
A \hat{H}_{1} B=\operatorname{conc}\left(A \hat{H}_{1} J\right)-J \hat{H}_{1} B=\left(\pi+O \hat{A} H_{1}\right)-\left(\pi-O \hat{B} H_{1}\right),
$$

since $\overline{A H_{1}}=\overline{K_{1} H_{1}}$ and $\overline{J H_{1}}=\overline{J B}$. Then equalities $O \hat{B} H_{1}=O \hat{B} A+A \hat{B} H_{1}$ and $O \hat{A} H_{1}=B \hat{A} O+H_{1} \hat{A} B$ yield:

$$
\begin{aligned}
A \hat{H}_{1} B & =\left(\pi+\left(B \hat{A} O+H_{1} \hat{A} B\right)\right)-\left(\pi-\left(O \hat{B} A+A \hat{B} H_{1}\right)\right) \\
& =(O \hat{B} A+B \hat{A} O)+\left(A \hat{B} H_{1}+H_{1} \hat{A} B\right)=(\pi-\alpha)+\left(\pi-A \hat{H}_{1} B\right),
\end{aligned}
$$

considering triangles $H_{1} A B$ and $O A B$. Using the first and last term we get $A \hat{H}_{1} B=\pi-\frac{\alpha}{2}$, i.e. $H_{1} \in \Gamma_{1}$.

Case 8-When point $H_{2}$ is on $J B$ - this time on $B$ 's side - we have (Fig. 42): 


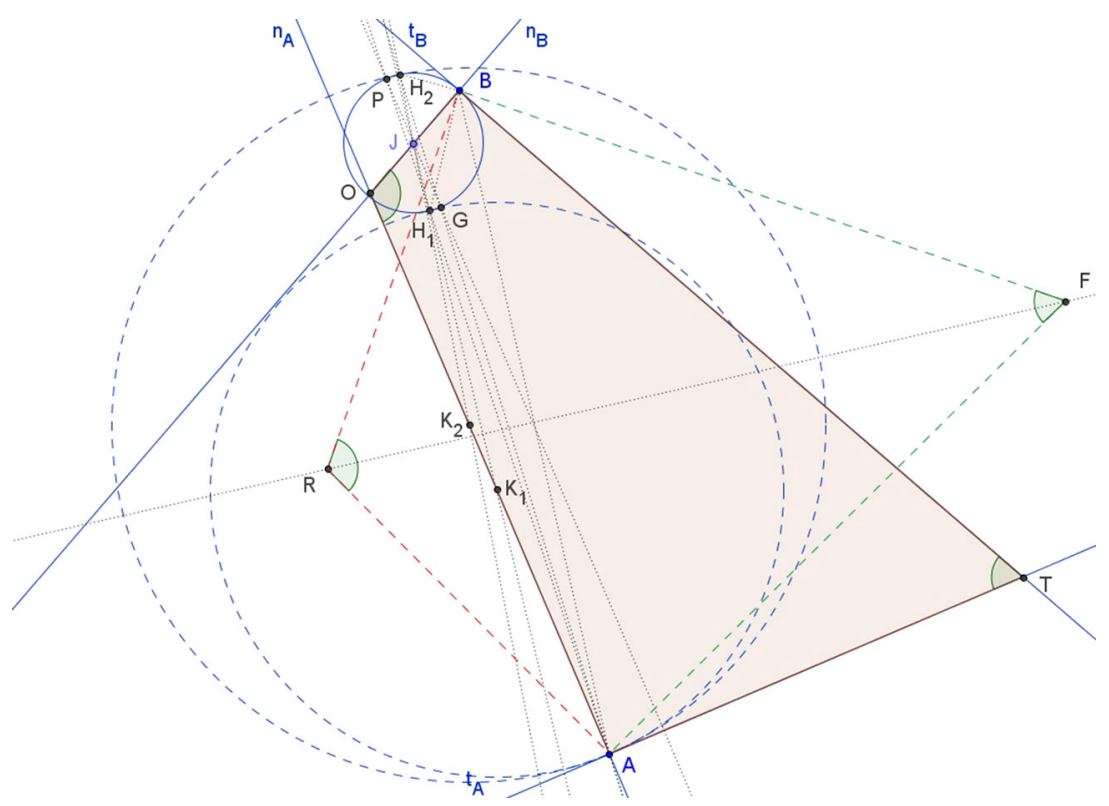

Fig. 40 Proving Theorem 7-Case 6

$$
A \hat{H}_{2} B=A \hat{H}_{2} J=O \hat{A} H_{2},
$$

since $\overline{A K_{2}}=\overline{K_{2} H_{2}}$. Then we use $O \hat{A} H_{2}=H_{2} \hat{A} B+B \hat{A} O$ and we get:

$$
\begin{aligned}
A \hat{H}_{2} B & =H_{2} \hat{A} B+B \hat{A} O=\left(\pi-\left(A \hat{H}_{2} B+H_{2} \hat{B} A\right)\right)+B \hat{A} O \\
& =\left(\pi-\left(A \hat{H}_{2} B+(\pi-O \hat{B} A)\right)\right)+B \hat{A} O=-A \hat{H}_{2} B+(O \hat{B} A+B \hat{A} O) \\
& =-A H_{2} B+(\pi-\alpha),
\end{aligned}
$$

considering triangles $H_{2} A B$ and $O A B$. Using the first and last term we get $A \hat{H}_{2} B=\frac{\pi-\alpha}{2}$, which proves that $H_{2} \in \Gamma_{2}$. For $H_{1}$ the proof is identical to that of Case 7.

Case 9-Point $\mathrm{H}_{2}$ is now on $A$ 's side w.r.t. $O B$. Both cases in Fig. 43 can be treated. Note that when $\Gamma_{4}$ is tangent to $T A$ there is only one circumference tangent to it. Both $H_{1}$ and $H_{2}$ are still on $F$ 's side w.r.t. $O A$. We have

$$
A \hat{H}_{2} B=A \hat{H}_{2} J-J \hat{H}_{2} B=O \hat{A} H_{2}-\left(\pi-O \hat{B} H_{2}\right),
$$

since $\overline{A K_{2}}=\overline{K_{2} H_{2}}$ and $\overline{J H_{2}}=\overline{J B}$. Then we use $O \hat{A} H_{2}=B \hat{A} O+H_{2} \hat{A} B$ and $\hat{O B} H_{2}=A \hat{B} H_{2}+A \hat{B} O$ getting:

$$
\begin{aligned}
A \hat{H}_{2} B= & \left(B \hat{A} O+H_{2} \hat{A} B\right)-\left(\pi-\left(A \hat{B} H_{2}+A \hat{B} O\right)\right) \\
= & \left(H_{2} \hat{A} B+A \hat{B} H_{2}\right)+(B \hat{A} O+A \hat{B} O)-\pi= \\
& \left(\pi-A \hat{H}_{2} B\right)+(\pi-\alpha)-\pi
\end{aligned}
$$




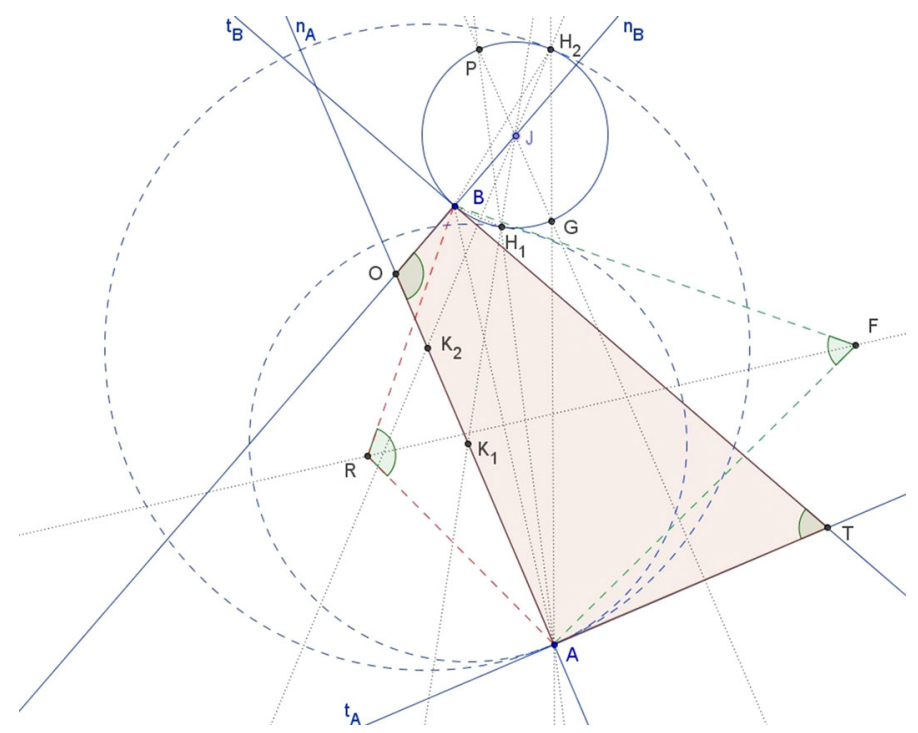

Fig. 41 Proving Theorem 7-Case 7

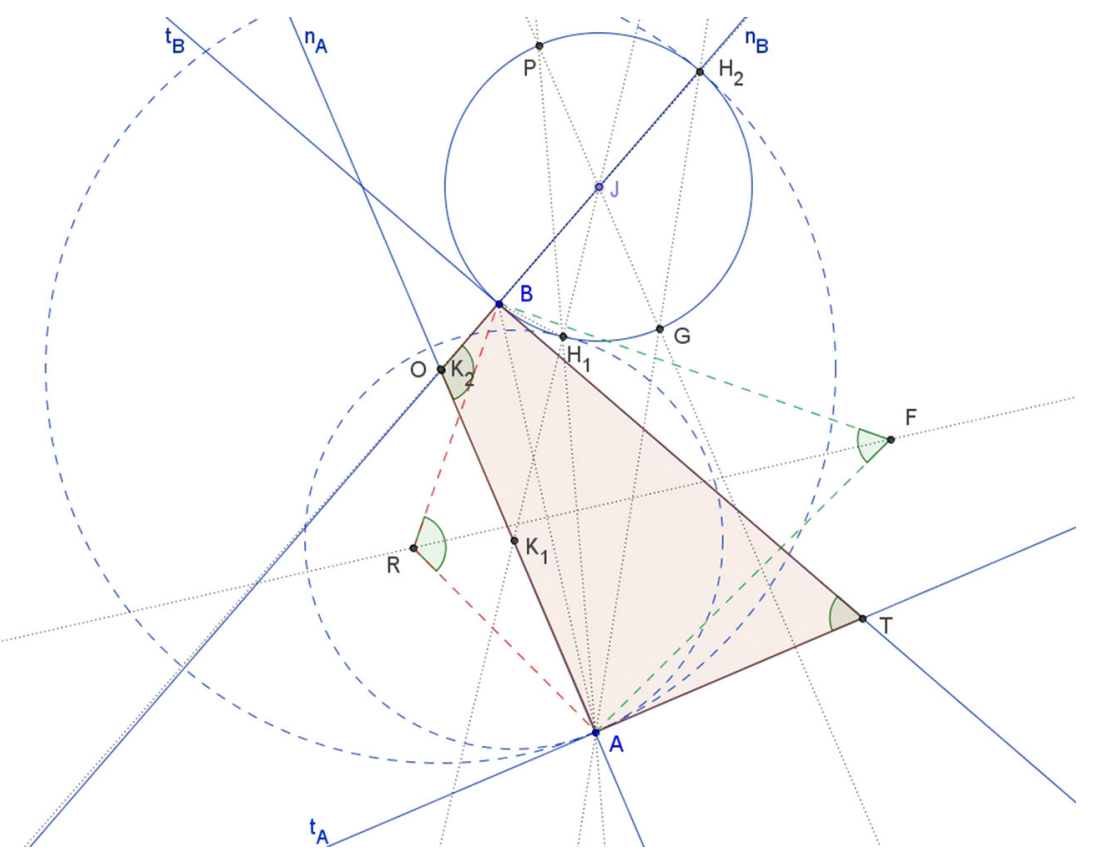

Fig. 42 Proving Theorem 7-Case 8 


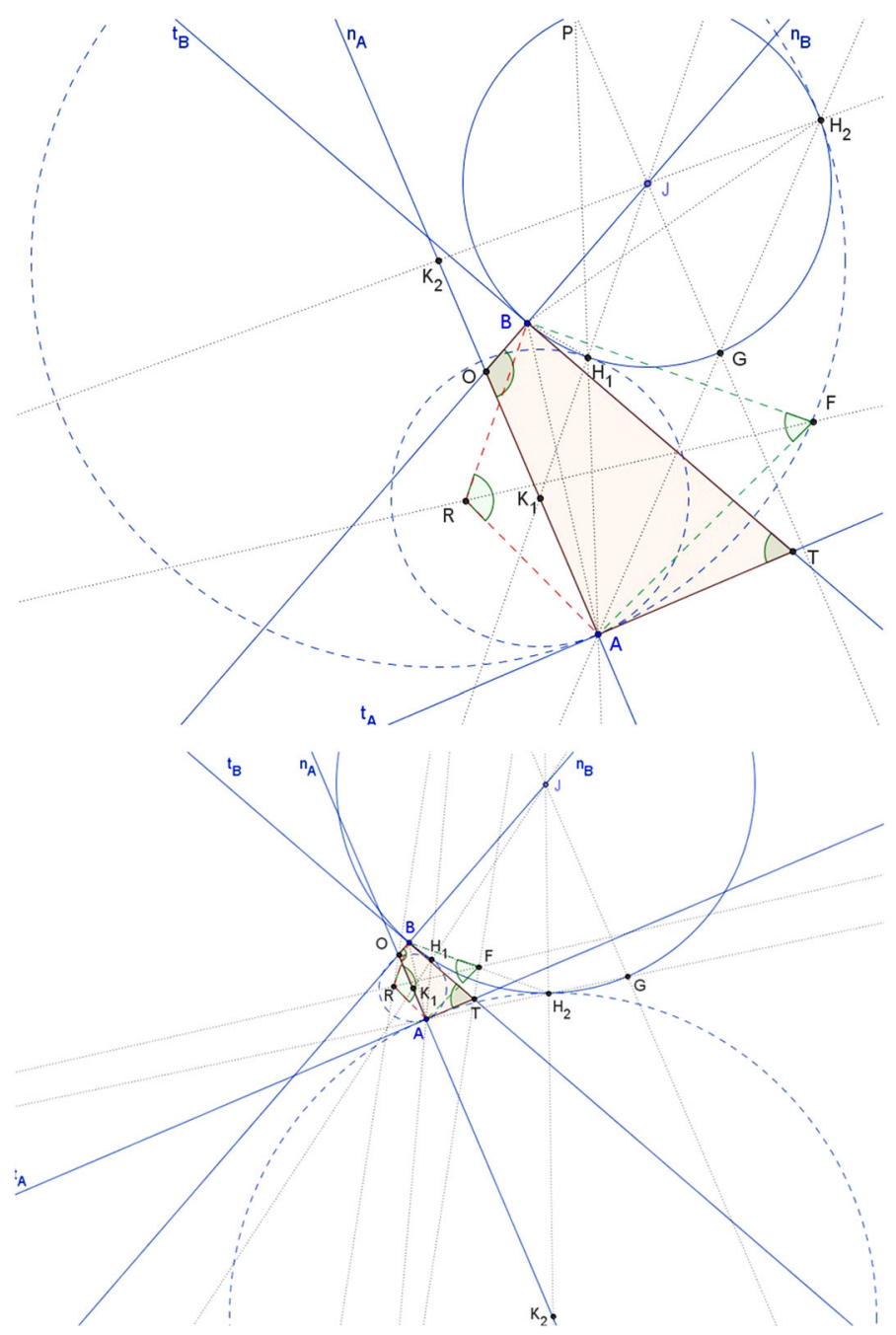

Fig. 43 Proving Theorem 7-Case 9

considering $H_{2} A B$ and $O A B$. Using the first and last term we get $A \hat{H}_{2} B=\frac{\pi-\alpha}{2}$, which proves that $\mathrm{H}_{2} \in \Gamma_{2}$.

For $H_{1}$ :

$$
A \hat{H}_{1} B=\operatorname{concave}\left(A \hat{H}_{1} J\right)-J \hat{H}_{1} B=\left(\pi+O \hat{A} H_{1}\right)-\left(\pi-O \hat{B} H_{1}\right),
$$

since $\overline{A H_{1}}=\overline{K_{1} H_{1}}$ and $\overline{J H_{1}}=\overline{J B}$. Then we use $O \hat{B} H_{1}=O \hat{B} A+A \hat{B} H_{1}$ and $O \hat{A} H_{1}=B \hat{A} O+H_{1} \hat{A} B$ and this yields:

$$
\begin{aligned}
A \hat{H}_{1} B & =\left(\pi+\left(B \hat{A} O+H_{1} \hat{A} B\right)\right)-\left(\pi-\left(O \hat{B} A+A \hat{B} H_{1}\right)\right) \\
& =(O \hat{B} A+B \hat{A} O)+\left(A \hat{B} H_{1}+H_{1} \hat{A} B\right)=(\pi-\alpha)+\left(\pi-A \hat{H}_{1} B\right),
\end{aligned}
$$




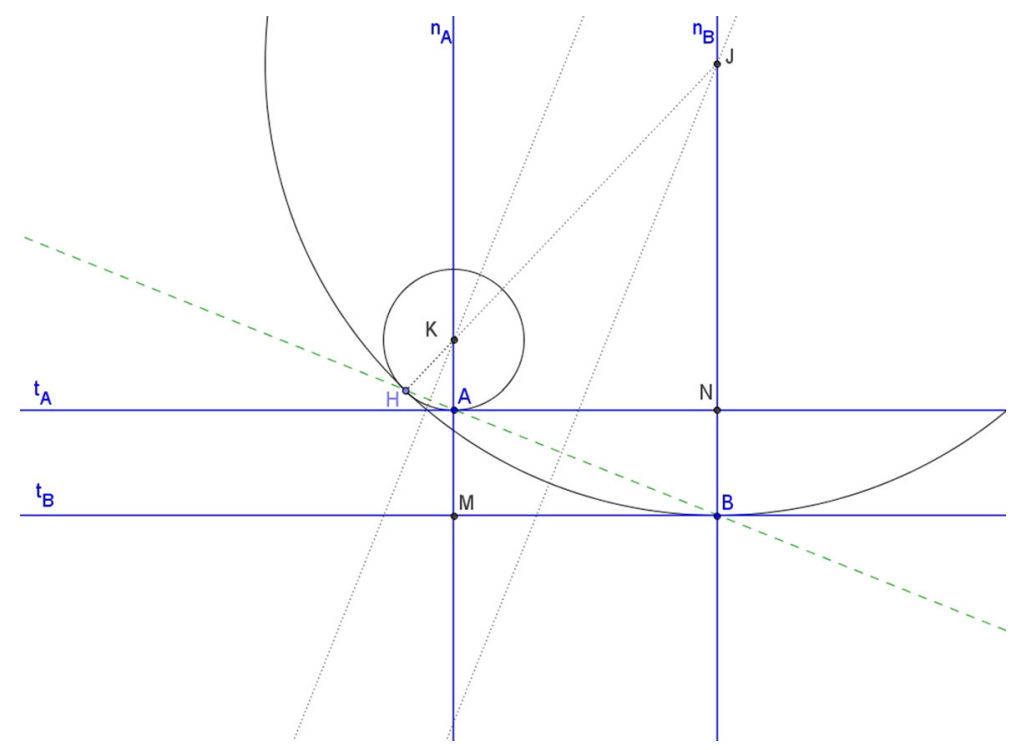

Fig. 44 Proving Theorem 9

considering $H_{1} A B$ and $O A B$. Using the first and last term we get $A \hat{H}_{1} B=\pi-\frac{\alpha}{2}$, which proves that $H_{1} \in \Gamma_{1}$.

The proof is now complete.

Proof of Theorem 9 We can use Fig. 44 as reference, keeping in mind that the proof remains exactly the same when $H$ is chosen otherwise on $A B$, or $J$ and/or $K$ happen to be on the other sides of $n_{B}$ and $n_{A}$ with respect to $A$ and $B$.

Take two circumferences tangent in $H$, one with centre $J$ on $n_{B}$, the second with centre $K$ on $n_{A}$ (clearly $H$ cannot coincide with neither $A$ nor $B$ ). Since $H \hat{K} A=H \hat{J} A$, because $n_{B}$ and $n_{A}$ are parallel and $H, K$ and $J$ are co-linear, we have that triangles $A K H$ and $B J H$, both isosceles, must have equal base angles $J \hat{B} H$ and $K \hat{A} H$, which implies for $H, A$ and $B$ to be co-linear.

On the other hand, taking $H$ on $A B-\{A, B\}$, and finding $K$ as the only possible centre on $n_{A}$ of a circumference through $H$ and $A$, and then $J$ as the intersection between $H K$ and $n_{B}$, we see that triangles $A K H$ and $B J H$ have the same angles, which implies that $B J H$ is also isosceles, and so $\overline{J B}=\overline{J H}$ and the circumference with centre $J$ and radius $\overline{J B}$ completes the pair.

\section{References}

Bosse, Abraham. 1655. Traité des Geometrales et Perspectives Enseignées dans l'Academie Royale de la Peinture et Sculpture. Paris: l'Auteur.

Dixon, Richard. 1987. Mathographics. Oxford: Basil Blackwell. 
Dotto, Edoardo. 2001. Note sulle costruzioni degli ovali a quattro centri. Vecchie e nuove costruzioni dell'ovale. Disegnare Idee Immagini XII 23:7-14.

Huerta, Santiago. 2007. Oval domes, geometry and mechanics. Nexus Network Journal 9(2):211-248.

López Moto, Ana. 2011. Oval for any given proportion in architecture: a layout possibly known in the sixteenth century. Nexus Network Journal 13(3):569-597.

Mazzotti, Angelo Alessandro. 2014. What Borromini might have known about ovals. Ruler and Compass Constructions. Nexus Network Journal. doi:10.1007/s00004-014-0190-z. (in this issue).

Ragazzo, Felice. 2011. Curve Policentriche. Sistemi di raccordo tra archi e rette. Roma: Prospettive.

Ragazzo, Felice. 1995. Geometria delle figure ovoidali. Disegnare idee immagini VI 11:17-24.

Serlio, Sebastiano. 1619. Tutte l'Opere d'Architettura et Prospettiva di Sebastiano Serlio [...]. Venezia: G. de' Franceschi.

Angelo Alessandro Mazzotti has a $\mathrm{PhD}$ in Operations Research (Dottore di Ricerca in Ricerca Operativa), and is a high school mathematics teacher and freelance mathematician. 